This manuscript is a preprint and will be shortly submitted for publication to a scientific journal. As a function of the peer-reviewing process that this manuscript will undergo, its structure and content may change.

If accepted, the final version of this manuscript will be available via the 'Peer-reviewed Publication DOI' link on the right-hand side of this webpage. Please feel free to contact any of the authors; we welcome feedback. 


\title{
Space-time susceptibility modeling of hydro-morphological processes at the Chinese national scale
}

\author{
Nan Wang ${ }^{1,2}$, Luigi Lombardo ${ }^{3}$, Weiming Cheng ${ }^{1,2,4,5, *}$, Mattia Marconcini ${ }^{6}$, \\ Felix Bachofer ${ }^{6}$, Liang Guo ${ }^{7,8}$, Junnan Xiong ${ }^{1,9}$
}

\footnotetext{
${ }^{1}$ State Key Laboratory of Resources and Environmental Information Systems, Institute of Geographic Sciences and Natural Resources Research, Chinese Academy of Sciences, Beijing, 100101, China

${ }^{2}$ University of Chinese Academy of Sciences, Beijing, 100049, China

${ }^{3}$ University of Twente, Faculty of Geo-Information Science and Earth Observation (ITC), PO Box 217, Enschede, AE 7500, Netherlands

${ }^{4}$ Jiangsu Center for Collaborative Innovation in Geographic Information Resource Development and Application, Nanjing, 210023, China

${ }^{5}$ Collaborative Innovation Center of South China Sea Studies, Nanjing, 210093, China

${ }^{6}$ Earth Observation Center, German Remote Sensing Data Center, Land Surface Dynamics, Oberpfaffenhofen, 82234, Wessling, Germany

${ }^{7}$ Research Center on Flood and Drought Disaster Reduction of the MWR, Beijing, 100038, China

${ }^{8}$ State Key Laboratory of Simulation and Regulation of Water Cycle in River Basin, China Institute of Water Resources and Hydropower Research, Beijing 100038, China

${ }^{9}$ School of Civil Engineering and Architecture, Southwest Petroleum University, Chengdu, 610500, China
} 
one, by considering four temporal cross-validation schemes (Forward-All, Forward-Sequence, Backward-All, and Backward-Sequence), removing the yearly multiple intercept. In the first of 31 temporal replicates, Forward-All is calibrated for 1985 and then used to predict from 1986 to 2015. In the second step, a model is calibrated for 1985 and 1986 combined and used to validate the rest of the space-time series. This is replicated up to the last model where the combined data from 1985 to 2014 is calibrated to predict the last year of the HMP presence/absence data. Forward-Sequence also moves in the same temporal direction but the sampling scheme sequentially extracts two years at a time, one for calibration and one for validation. For instance, the first step is trained for 1985 and used to predict 1986; then the second step is trained for 1986 and used to predict 1987. As for Backward-All, and Backward-Sequence, their structure is the same but the temporal direction goes from 2015 to 1985.

Our explanatory model suggests that the overall number of HMP events per year has increased in the last decade and that the annual susceptibility has subsequently followed the same trend. As for the four cross-validation routines, Forward-Sequence shows excellent performance with an average AUC of 0.83, slightly better than Forward-All, BackwardAll, and Backward-Sequence. From an interpretative standpoint, this implies that the best spatio-temporal prediction we obtained is associated with short-term variations of the HMP distribution and that such variations should be considered in a forward temporal direction.

Furthermore, we portrayed the annual susceptibility models into 30 maps, where the south-east of China is shown to exhibit the largest variation in the spatio-temporal probability of HMP occurrence. Also, we compressed the whole spatio-temporal prediction into three summary maps. These report the mean, maximum and $95 \%$ confidence interval of the spatio-temporal susceptibility distribution per catchment, per year.

The information we present has a dual value. On the one hand, we provide a platform to interpret environmental effects on HMP at a very large scale, both spatially (the whole Chinese country) and temporally (31 years of records). On the other hand, we provide information on which catchments are more prone to experience a HMP-driven disaster. Hence, a step further would be to select the more susceptible catchment for detailed analysis where physically-based models could be tested to estimate the potentially impacted areas.

Keywords: Hydro-morphological processes; Historical hazard archives; Susceptibility; Spatiotemporal predictive models. 


\section{Introduction}

In this work, the term hydro-morphological process (HMP) was used to address a class of earth surface phenomena where solid and fluid phases of a gravitationally-driven moving mass are not well determined. Thus, in this class we refer to a broad spectrum of processes in between debris flows and flash floods. The reasons behind such initial disclaimer are due to the nature of the dataset we used and further explanations will be provided later in the text.

This class of HMPs includes some of the most frequent and damaging natural disasters, and their occurrence shows a close relationship with climatic changes (Blöschl et al., 2020; Prein et al., 2017; Westra et al., 2014). Because of this, HMPs have increasingly been reported to threaten human lives and infrastructure in recent years (e.g., Špitalar et al., 2014). To prevent or limit the losses, it is crucial to estimate where and when these processes may occur. In turn, this enables administrations to plan ahead and mitigate future risks (Lombardo et al., 2020; Rossi et al., 2019).

HMPs are extremely rapid phenomena. Just few hours are needed between the triggering heavy rain and their manifestation (Borga et al., 2007; Marchi et al., 2010). They can also be generated by snow-melt but it is generally the intensity and duration of precipitation that control the process through the water discharged over a given area. Then, the overland flows follow the river network, entrains all sorts of debris and leaves it strewn especially when the runoff intersects urban areas (Norbiato et al., 2008). In such cases, roads may be blocked, drainage systems clogged, cars trapped, lives lost and property destroyed (Karagiorgos et al., 2016; Mahmood et al., 2017). For this reason, HMP prediction models are primarily implemented in a physically-based framework where one can reliably introduce the rainfall input and simulate the process by accounting for topography and soil hydrological characteristics (Tramblay et al., 2010). This is usually performed specifically for small areas (Rozalis et al., 2010) but recent advancement have led to develop similar applications on much wider regions, simulating different types of HMPs from catchment (Javelle et al., 2010; Bout et al., 2018) to country-wise scales (Gourley et al., 2017), and even up to continental scales (Paprotny et al., 2017). These different levels of details all share a common structure where a design storm is used as the input. The design storm can be either inferred from long time-series of rainfall data via extreme value statistics ( $\mathrm{Li}$ et al., 2019a). Or, it can be directly plugged in by using near-real-time rainfall data obtained from meteorological forecasts (Collier, 2007). As for the remaining information, terrain characteristics are commonly derived from global DEM data (Adnan et al., 2019) or from site-specific LiDAR surveys (Crema et al., 2018). Besides, soil parameters are required to describe the hydrological characteristics and the associated ability to retain water or to convert it into runoff (Norbiato et al., 2008). This can be obtained via in-situ tests whenever the area is relatively small (Cenci et al., 2016) and from global estimates such as ISRIC, for large scale assessments (Ragettli et al., 2017). These methods have the inherited ability to produce HMP runout estimates, such as total impacted spatial extent, flow heights, kinetic energy, volumes and more, which are crucial 
information for engineering design and master plans (Li et al., 2019b). However, the applicability of physically-based models inevitably suffers from considerable limitations whenever the study target involves continental to global scales (Bout et al., 2018; Glade and Crozier, 2005), with very few exceptions to this rule (Liao et al., 2012). In fact, for large areas, the required input information is typically quite smooth, assuming it is even accessible. And, collecting suitable geotechnical data is difficult if not impossible (Gaume et al., 2009) over large regions. As a result, a complementary branch in the natural hazard community has developed statistically-based models during the last decades. This methods do not offer the same breath of results produced from the physically-based counterpart (e.g., they do not spatially predict runout-impacted areas nor flow-heights, etc.). However, they provide useful information on areas potentially subjected to HMPs, learning from past events from which spatio-temporal projections are made (Gourley et al., 2013).

The present work fits in the second category. Specifically, the Chinese government has recently completed a long lasting project where all the available information on historical HMPs has been collated for the whole Chinese territory. We use the term HMP specifically because the Chinese catalogue reports a wide spectrum of earth surface processes without explicitly attributing a class. This catalogue starts from reports gathered even from ancient China and it covers the period until 2015. Because of this wide temporal coverage, the data differs in quality across space and time and the Chinese government has decided to use a more general classification, consistent through time. More specifically, the data collated until 1949 is relatively poor and the situation improves substantially from 1950 onward as the current Chinese government was established. Nevertheless, even from 1950 up to 1980, the data may still have some positional issues because the digital system did not exist (Li et al., 2018). The Chinese HMP report system became standardized after the 1980ies, with more available technologies being used to record the location (latitude and longitude), date and time as well as the losses expressed either in the number of victims or economical value (Guo et al., 2018). In light of this considerations, we subset the Chinese HMP catalogue extracting all the available information from 1985 to 2015. We note here that since 1985 we also have access to meteorological digital data collected and aggregated daily from the Chinese rain gauge network.

We use this data to build a space-time HMP susceptibility model. A susceptibility model essentially estimates the probability of occurrence of a given natural process within a specific mapping unit and temporal unit. Mapping units constitute the spatial structure under which a given study area is subdivided. They can consist of a regular lattice (usually grid-cells or rarely hexagons) or they can represent geographic features such as catchments or administrative units (Carrara et al., 1991, 1995; Reichenbach et al., 2018; Lombardo et al., 2019). Irrespective of the specific geometry, a mapping unit represent the object upon which a statistical model estimates the probability of occurrence of the target hazard. As for temporal units, this represent the time span upon which the selected model makes a prediction. For physically-based models, this is typically expressed in hours or days whereas 
for statistical models this may involve a much larger time span. In this work we opted for a catchment partition, having accessed the most updated watershed delineation of China (Shen et al., 2017). As for the temporal partition, we selected an annual unit of time.

As for the method, we chose a binomial Generalized Linear Model (GLM) assuming that the spatio-temporal population of HMPs across China behaves according to a Bernoulli probability distribution. This procedure is quite common and actually represents the most common practice in the geomorphological literature (e.g., Budimir et al., 2015; Lombardo et al., 2015; Reichenbach et al., 2018).

We stress here that the susceptibility to any surface process is not stationary or timeinvariant (Lombardo et al., 2020). It actually varies through time as the environmental conditions change. For instance, landscape evolution processes may modify the terrain, hence changing the hydrology of a given area. Similarly, settlement growth and urbanization experienced a dynamic expansion and the urbanization itself has become denser through time, especially in China. This may have changed the distribution of permeable surfaces in favor of concreted and sealed land covers (see, Gong et al., 2019). Also, climate changes may contribute to vary the HMP triggering conditions through space and time, especially because rainfall regimes have become less diluted during wet seasons and they have become more concentrated in narrow time windows. All these contributing/triggering factors can be accounted for in statistical models. For instance, if climate change and accelerated urbanization control the HMP occurrence distribution, then a space-time statistical model should be able to capture their influences and show a potential increase in HMP occurrences in recent years.

The present manuscript is organized as follows: Section 2 introduces the study area and Section 3 describes the material and methodology framework used in susceptibility modelling. This is followed by a detailed description of the model performance and the resulting susceptibility maps in Section 4. Finally, Section 5 discusses the supporting and opposing arguments on this work. And Section 6 summarizes our final remarks.

\section{Study Area}

China approximately covers the area between latitudes $18^{\circ}$ and $54^{\circ} \mathrm{N}$, and longitudes $73^{\circ}$ and $135^{\circ} \mathrm{E}$. It is characterized by a vast territory and a complex landscape. Based on geomorphological characteristics, China can be divided into six homogeneous regions (Wang et al., 2020): eastern plains, southeastern hills, southwestern mountains, north-central plains, northwestern basins and Tibetan Plateau. About two-thirds of China is covered by mountainous areas (Liu et al., 2018). The southern China consists of hilly and mountainous terrains, while the western and northern China is dominated by plains and basins. The annual rainfall records are strongly controlled by the distance to the coastline and precipitation amounts gradually decrease from the southeast to northwest of China. The eastern plains and southern coasts are severely influenced by the East Asian Summer Monsoon, where most 
of China's agricultural land and settlements are located. In this context, only the northwest China has a predominantly arid climate and a lower population density.

\section{Material and Methods}

\subsection{Hydro-morphological processes in China}

As previously introduced, the Chinese catalogue of HMPs is a digital collection of events, describing a spectrum of phenomena where a fast moving mass - consisting of a ill-defined proportion of solid and fluid - propagates across the landscape, potentially causing destruction in its path. As a result, the above mentioned spectrum encompasses processes from debris flows (where the solid and liquid phases are almost equally represented) to flash floods (where the fluid phase is much larger than the solid one). Each HMP record in the database contains information on geographic coordinates, date and time as well as (but not always) two loss estimates, expressed as victims and costs.

Because of this rich information, it would be theoretically possible to extract HMPs that have actually resulted in a disaster (i.e., life losses $>0$ OR economical losses $>0$ ). However, not all the HMPs contain the loss information. For this reason, instead of modeling a subset of the whole database, we opted for the entirety of the available information, including "innocuous" and disastrous HMPs. This information is geographically summarised in Figure 1 where we highlight the spatio-temporal distribution of HMPs upon which we have built our modeling routine.

Overall, the Chinese database reports 24,956 HMPs in the time span of 31 years (19852015 ) with a substantially varying concentration across space and time, with the exception of the western arid to semi-arid sector where essentially no events have been recorded.

\subsection{Mapping unit}

The nature of the Chinese HMP catalogue implies that the various processes included may act on different spatial scales. For instance, debris flows usually have a more limited spatial extent, thus slope- to catchment- based models are the most suitable to represent the physical expression of these phenomena. Conversely, on the other side of the spectrum, flash floods can travel much longer distances, therefore covering larger geographic scales and associated models, from slope to regional ones. Because of this, choosing the most appropriate mapping unit becomes a crucial step to handle the spatio-temporal dimension of the HMP data. We recall here that a mapping unit, in its most basic form, represents the geographic object upon which the landscape is partitioned. In case of relatively small study areas, examples exist where HMPs are modeled along specific streamlined and neighboring areas by adopting a fine squared lattice. This type of resolution and characterization of the HMPs cannot be used in our case, where the size of the Chinese territory would result in billions of grid-cells or data points. Therefore, in case of such large geographic context, a common spatial partition choice 


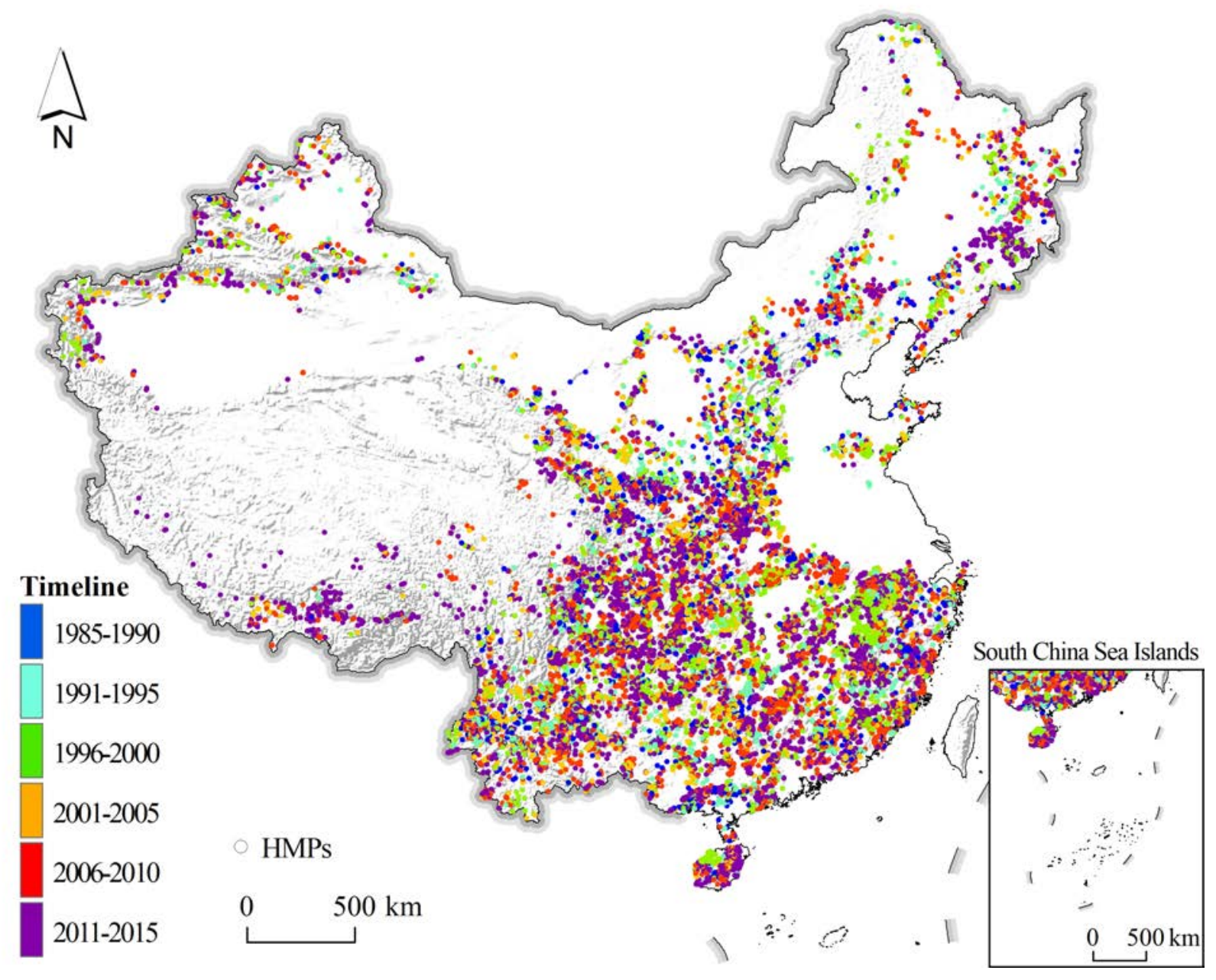

Figure 1: The multi-temporal HMPs in China from 1985 to 2015. 
could be represented by administrative boundaries, upon which estimating the probability of HMP occurrences. However, the resulting susceptibility model would neglect the hydrology behind the natural process. In fact, administrative boundaries do not necessarily follow streams or catchment divides, where HMP occurrences can be considered independent or nearly-independent from each other. Therefore, a good solution to represent the spatial scale of HMPs, while respecting the hydrological realization of the natural phenomena, is to consider a catchment partition of the Chinese territory. To support the analyses in this work, we selected the $12^{\text {th }}$ level catchment delineation published by Shen et al. (2017), which partitions the whole Chinese territory into 73,587 catchments. The corresponding distribution of catchment sizes is bimodal (see Figure 2) and it spans from $0.1 \mathrm{~km}^{2}$ to $667 \mathrm{~km}^{2}$, with average area of $130 \mathrm{~km}^{2}$ and a $95 \%$ confidence interval - measured as the difference between the 97.5 and the 2.5 percentiles of the distribution - of $231 \mathrm{~km}^{2}$.

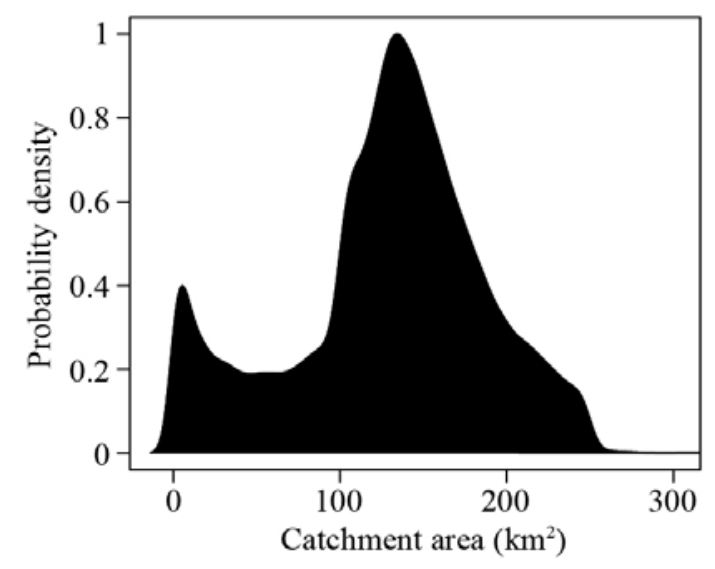

Figure 2: Probability density distribution of catchments sizes in China, computed from the $12^{\text {th }}$ level published in Shen et al. (2017).

As any other mapping unit partition used the context of susceptibility modeling, a preprocessing step is required. The presence/absence information of HMPs is to be assigned to each catchment. To do so, we assign a presence (1) and absence (0) label to catchments where at least one HMP record is contained within a specific temporal unit (see details below).

\subsection{Temporal unit}

As much as the mapping unit choice aggregates HMP occurrences over space, whenever a dataset has a temporal connotation one should also choose a temporal unit. A temporal unit is the time interval through which we aggregate HMP occurrences and assign a suitable presence/absence conditions. In our case, the HMP dataset has very fine resolution with date and time available. However, the properties or covariates we will use in the model (see Section 3.4) do not share the same temporal resolution. For instance, rainfall and temperature are 
available with a daily resolution across China, vegetation cover and urban development are available on a yearly basis while terrain properties do not exhibit any temporal changes. Therefore, choosing a timescale that allows for meaningful interpretation and suitable data is also crucial. In this context, the coarse temporal resolution of the covariates inhibits our ability to build a finely resolved space-time model. And, in any case, choosing a fine temporal resolution would inevitably increase the computational burden. Thus, choosing a reasonable trade-off is required. Due to the characteristics of some crucial covariates, we chose a yearly temporal unit. Such temporal unit implies that we assign a presence (1) and absence (0) label to catchments where at least one HMP record is contained within a year time window.

\subsection{Covariate set}

HMPs are the result of several interplaying factors. These primarily feature:

1. precipitation, for it represents the main trigger;

2. catchment morphology, for it controls the time of concentration and other hydrodynamic parameters;

3. terrain attributes, for they control the path of the overland flows as well as the availability of material to be mobilized and transported;

4. soil hydrology, for it controls the interaction of the water with the earth surface;

5. vegetation density, for it can absorb part of the rainfall discharge and interact with soil through the root system;

6. temperature, for it controls evapotranspiration and hence the soil moisture;

7. urbanization, for it may change the natural hydrology both because of impermeable surface placed over permeable ones, and because buildings can also reduce the hydraulic section through which HMPs may flow into.

In the context of space-time modeling, these properties need to be considered both in terms of their spatial distribution and also in terms of their temporal evolution. In fact, some properties will be more stationary over time, whereas some will have a much more rapid rate of change. For instance, at the scale of the Chinese territory, soil hydrology can be considered quite stationary within the 31 years under consideration. Conversely, rainfall, vegetation and urbanization have a much faster spatio-temporal variation. Therefore, certain properties can be introduced as a single realization (or map) whereas other properties should be accounted for their successive temporal realizations (or maps).

We also consider antecedent HMPs, calculated over a time window of three years and binarized into presence/absence conditions per catchment. We do so, to carry the spatial signal of the HMPs. In fact, within a relatively short time window, we expect the susceptibility to HMPs to be quite spatio-temporally consistent or stationary. In other words, areas that have experienced HMPs in the recent past are more likely to suffer from HMP events in the near future (Samia et al., 2017). Hence, introducing the information of previously 
occurred HMPs should better inform the model of this short-term spatial dependence and improve its overall prediction capacity (Lombardo et al., 2020).

As much as we tried to capture some residual dependence over space via antecedent HMP events per catchment, we also tried to consider the presence of residual temporal dependencies. Our assumption is that if climate change has produced a increasing trend in rainfall extremes and resulting HMPs, a multiple intercept should also show an increasing contribution through time.

The modeling protocol we implemented makes use of both types of covariates, featuring properties that can be safely considered time-invariant within three decades: terrain and catchment characteristics as well as soil type and climatic zones. And, also by featuring properties that are explicitly time-variant within the same period: climate, vegetation and human activity, as well as antecedent HMP events).

Due to the size of the study area and the temporal connotation of the database, the number of covariate is inevitably large especially because a crucial step consists of aggregating the covariate values in space (at the catchment scale) and time (at the yearly scale). Due to the numerous data sources, the spatial resolution of the covariate set we chose ranges from $90 \mathrm{~m}$ (SRTM, https://earthexplorer.usgs.gov/) to $8 \mathrm{~km}$ (NDVI, https://climatedataguide.ucar.edu/). To summarize the spatial signal of each covariate (per catchment) we calculated its mean and standard deviation. In case of stationary covariates, such as terrain attributes, the spatial mean and standard deviation is a sufficient approximation where the mean reflects the main bulk of the pixel distribution per catchment and the standard deviation highlight the associated variability. These values are kept constant through time. As for catchment morphological indices, one single value is computed per catchment and even in this case, the indices are kept constant through time (they are repeated for each of the 31 years).

For covariates that are nonstationary over time (such as rainfall, temperature and vegetation) we compute the spatial mean per catchment as well as he temporal mean and standard deviation in a year. As for the anthropic signal, the percent of urbanized area with respect to the total catchment size is directly calculated on a yearly basis, hence it does not need any spatio-temporal aggregation. To this purpose, we employed the World Settlement Footprint (WSF) Evolution which outlines at $30 \mathrm{~m}$ spatial resolution the global settlement growth from 1985 to 2015 on a yearly basis (Marconcini et al., 2020a). The WSF evolution has been generated by exploiting the recently released WSF2015 layer, which maps worldwide the settlement extent for the year 2015 (Marconcini et al., 2020b). In particular, for each pixel denoted as settlement in the WSF2015, a temporal analysis has been performed by means of historical Landsat-5 and Landsat-7 optical satellite imagery to identify when the construction took place. Here, an iterative procedure has been implemented where - starting backwards from 2015 - training samples for the settlement and non-settlement class are extracted out of the map obtained at time $t$ and Random forest binary classification has been employed to outline the settlement extent at time t-1. Ultimately, zonal statistics have been 
computed to determine yearly for each catchment partition the corresponding total amount of settlement area.

A summary of all the covariates we considered is provided in Table 1.

\subsection{Susceptibility Modeling}

In this work, because of the vast study area and the long time series, we opted to create a susceptibility model that can feature spatio-temporal characteristics. We do so by considering two types of models, an explanatory one and a set of predictive ones. The explanatory model is a model built by using the whole available information. In our case, it is a model where the entirety of China is taken into consideration together with its 31 years observations. In such a way, one can build a model that can be used for interpretation, to understand the statistical role of every environmental factor with respect to HMP occurrences. However, such models do not have a predictive connotation because no new data is used to test the classification performance. In fact, predictive models are built by calibrating the analysis over a portion of the data. And, the calibrated relations are used to make a prediction over an unknown dataset.

We stress here that the natural hazard community - at least the part of it using statistical models - usually performs calibration by randomly subsetting a percentage of the data over space and test the validation performance over the complementary cases. However, prediction or forecast are terms usually referred to estimates of future occurrences, hence in time. Rarely, studies dedicated to susceptibility models are validated in time (or chronovalidated) (Lombardo and Tanyas, 2020; Cama et al., 2015), mostly because of the inherited complexity of obtaining accurate multi-temporal inventories (Guzzetti et al., 2012).

Because our dataset spans over such a large time window, we actually have the chance to test whether it is possible to forecast future occurrences. Thus, we have opted to assess the predictive capacity of future HMP occurrences by considering four cross-validation schemes:

1. Forward-All or MOD1: This validation procedure starts by calibrating our binomial GLM (more details in Section 3.5.1) over a specific year (e.g., 1985) and testing over the remaining time series (e.g., 1986-2015). In the second step, the previous reference year is combined with the next (e.g., 1985 and 1986) to predict HMPs in the remaining years (1987 to 2015). This moving window moves one year at a time until completion of the time series.

2. Forward-Sequence or MOD2: This validation scheme iteratively calibrates over a specific year (e.g., 1985) and predicts only the next (e.g., 1986). In the second step, the calibration aggregates the subsequent year (e.g., 1985 and 1986) and predicts only the next (e.g., 1987). This is repeated until the completion of the time series in 2015.

3. Backward-All or MOD3: This validation scheme is analogous to MOD1 but it is implemented in the opposite temporal direction. Specifically, we calibrate over the last year 
Table 1: Covariates' summary: (Time-invariant variables: terrain feature, stream/catchment feature, soil type, and climatic zone; Time-variate variables: climatic indicators, NDVI, settlement area, 3-years antecedent HMP events.

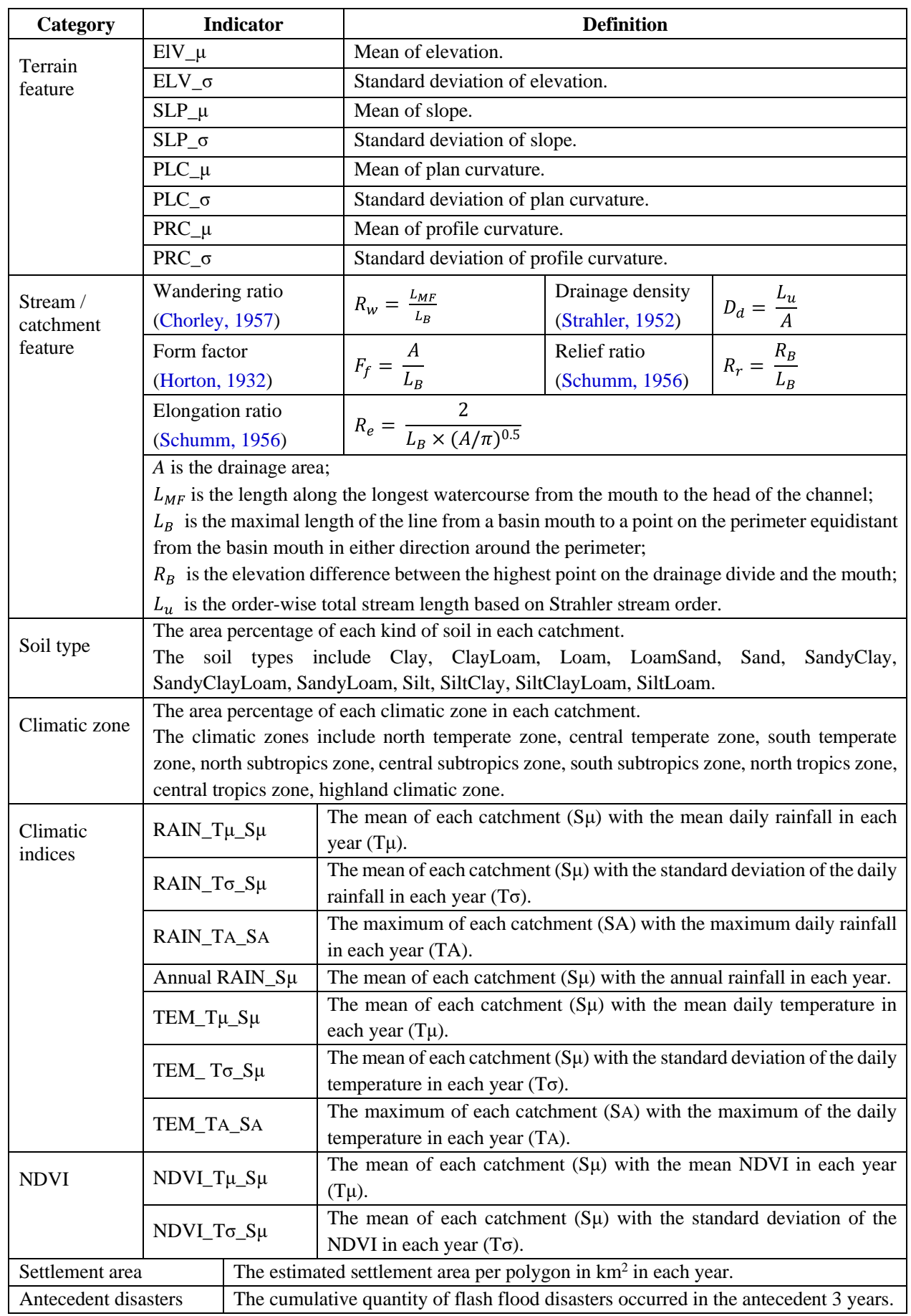


(2015) and predict the whole time series backward (from 1985 to 2014). In the next step we then calibrate aggregating the information of the previous year (e.g., 2015 and 2014) to predict the remaining time series (1985 to 2013). This operation is backwardly repeated until the completion of the time series in 1985.

4. Backward-Sequence or MOD4: this model is analogous to MOD2 but again it is implemented in the opposite temporal direction. This means that the calibration starts in 2015 and it is used to predict the previous year only (2014). Then the calibration integrates the information from the previous year (2015 and 2014) to predict only one step back in time (2013). This operation is repeated backwardly until the time series is completed in 1985 .

Notably, each of these validation schemes inevitably produces 30 testing outputs, whereas the explanatory model only produces one training output.

\subsubsection{Generalized Linear Models}

The vast majority of statistically-based susceptibility models are carried out by using Generalized Linear Models (Budimir et al., 2015; Reichenbach et al., 2018). This class of models assumes that the response variable follows an exponential family distribution such as Gaussian, Poisson, Bernoulli and more. Among those, the Bernoulli case, also referred to as Binary Logistic Regression, corresponds to a model where the target variable can take on only two values. Therefore, a binomial GLM estimates the probability that a given mapping unit belongs to one of the two classes (by standard, this is the class 1, or the class conveying the presence of HMPs, rather than 0). More specifically, a binomial GLM can be denoted as follows:

$$
\operatorname{logit}(\pi)=\frac{\pi}{1-\pi}=\beta_{0}+\beta_{1} X_{1}+\beta_{2} X_{2}+\cdots+\beta_{n} X_{n}
$$

where, the target variable $Y$ is assumed to be Binomial with a probability $\pi$ of a given catchment to experience a HMP. The $\beta_{0}$ term is the global intercept and $\beta_{n}$ are the regression coefficients estimated for $X_{n}$ covariates. The logit, or the natural logarithm of the odds, allows for the conversion of the odds into probabilities.

This framework allows for continuous and discrete covariates. Each class of a discrete covariate is modeled independently from the other classes, or technically it is assumed to be independent and identically distributed (iid). More specifically, the model will assign a different regression constant to each class separately from the others. Notably, in this work we make use of iid covariates for a multiple yearly intercept for the explanatory reference model. The remaining covariates are all continuous in nature and used as linear properties both in the explanatory and predictive models. 


\subsubsection{Estimates of confidence intervals}

In statistics, any model should allow for inference on a distribution of estimates rather than a single estimate. In other words, obtaining a mean prediction is as important as measuring the uncertainty around that mean value. Therefore, in this work we sought to retrieve both the mean behavior of every regression coefficient and performance metric as well as the estimated variability associated with them.

To do so, we present two schemes, one for the explanatory model and one for the validation routines (MOD1 to MOD4). When implementing the explanatory model (we recall here that it is fitted using the whole available information), we have also added a bootstrap simulation step (Efron and Tibshirani, 1994). This step essentially re-samples with replacement the whole dataset and re-fits the same model structure to the simulated dataset. We do this over 100 bootstrap replicates to estimate the sampling distribution of each parameter we store during the explanatory analyses. Besides, we implement the 10-fold cross validation to evaluate the overall performance on the whole dataset. As for the validation routines in MOD1 to MOD4, the variability of the tests is summarized via the 30 estimates, one for each of the 30 years under consideration.

\subsubsection{Model evaluation}

The primary tool to assess the performance of our HMP susceptibility model consists of the Receiver Operating Characteristic curves (ROC, Hosmer and Lemeshow, 2000) and their integral or Area Under the Curve (ROC, Hosmer and Lemeshow, 2000). The former is the most common threshold independent metric used in classification problems (Rahmati et al., 2019). It is constructed by slicing the probability spectrum at various cutoff, and by computing the confusion matrix at each step. As a result, it is possible to calculate the False Positive Rate or FPR (FP / [FP+TN]) and the True Positive Rate or TPR (TP / [TP+FN]) for each cutoff. The integral of the curve defined by the FPR and TPR pairs calculates from different cutoffs can be then used as an index of performance. Specifically, $A U C=1$ indicate a perfect classification, $0.9<A U C<1$ refers to outstanding performance, $0.8<A U C<0.9$ marks excellent performance whereas $0.7<A U C<0.8$ are acceptable results. Any $A U C$ value from 0.7 to 0.5 indicates a range of poor performance down to results comparable to a random classification.

We make use of the AUC throughout the manuscript. We also implement a Jackknife test in the validation scheme (Lombardo and Mai, 2018; O'Banion and Olsen, 2014). A Jackknife test is essentially divided into two steps. The first one runs single $\left(j^{\text {th }}\right)$ variable models whereas the second runs all-but-one-variable $(j-1)$ models. In both cases, the AUC is calculated to offer a comprehensive summary of covariates contributions. Single variable models highlight stand-alone performance of specific covariates in explaining HMP occurrences. All-but-one-variable models highlight performance drop resulting from the removal of one single covariate at a time, with respect to a full model using them all at once. 
Notably, the validation scheme in this work includes training and testing 30 temporal models. As a result, we have run 30 Jackknife tests, one for each year from 1985 to 2015.

\section{Results}

\subsection{Explanatory Model and its cross-validation}

In this section, we reported the regression coefficients obtained from a susceptibility model built by using all the available spatio-temporal information. These estimates were used to interpret the relation between HMP occurrences and environmental conditions (or covariates). Firstly, each regression coefficient is characterized by a distribution of values which have been retrieved from 100 nonparametric bootstrap replicates. Figure 3 summarizes each model component. Among the continuous covariates (see Fig.3), climatic indices (e.g. $R A I N_{-} T \sigma_{-} S \mu$, AnnualRAIN_S $\mu$ ), terrain attributes (e.g. PLC_ $\sigma, S L P_{-} \sigma$ ), catchment morphology (e.g. form factor) present notable positive regression coefficients. In addition, catchments located in Central temperate and South temperate zones also suffer more from the HMPs. More details on the interpretation of this covariate effects will be provided in Section 5.

Besides, we made use of an iid effect for each year, whose result is shown in Figure 4. The year-specific regression constants show an interesting pattern. For each year from 2002 to 2014, all regression coefficients are significantly positive and the whole distribution is quite distant from the zero line (between 0.5 and 1) with an exception of 2004. As for each year in the period between 1985 and 2001, the regression constants are also estimated with a positive median coefficient, although some of them appear to be not significant (the distribution of regression constants also show negative values). Besides, the regression coefficients vary around the zero. More details on the interpretation of this temporal iid effect will be provided in Section 5.

to complete the analyses on the whole spatio-temporal domain, we also run a 10-fold cross validation. We recall here that a 10 -fold cross validation implies randomly partitioning the whole data population into ten complementary subsets, each time extracting $90 \%$ and $10 \%$ for calibration and validation, respectively. Figure 5 presents the performance of the 10-fold cross-validation scheme. Specifically, panel 5a reports 10 ROC curves obtained by using $90 \%$ of the spatiotemporal HMP data; and panel $5 \mathrm{~b}$ reports ROC curves obtained by testing over $10 \%$ of the spatiotemporal HMP data. The respective mean AUC values do not significantly change, as they both returned 0.84 . This attest both for excellent goodnessof-fit and prediction-skill according to Hosmer and Lemeshow (2000) as well as a indicating robust results with differences that can be distinguished only at the third decimal place. 


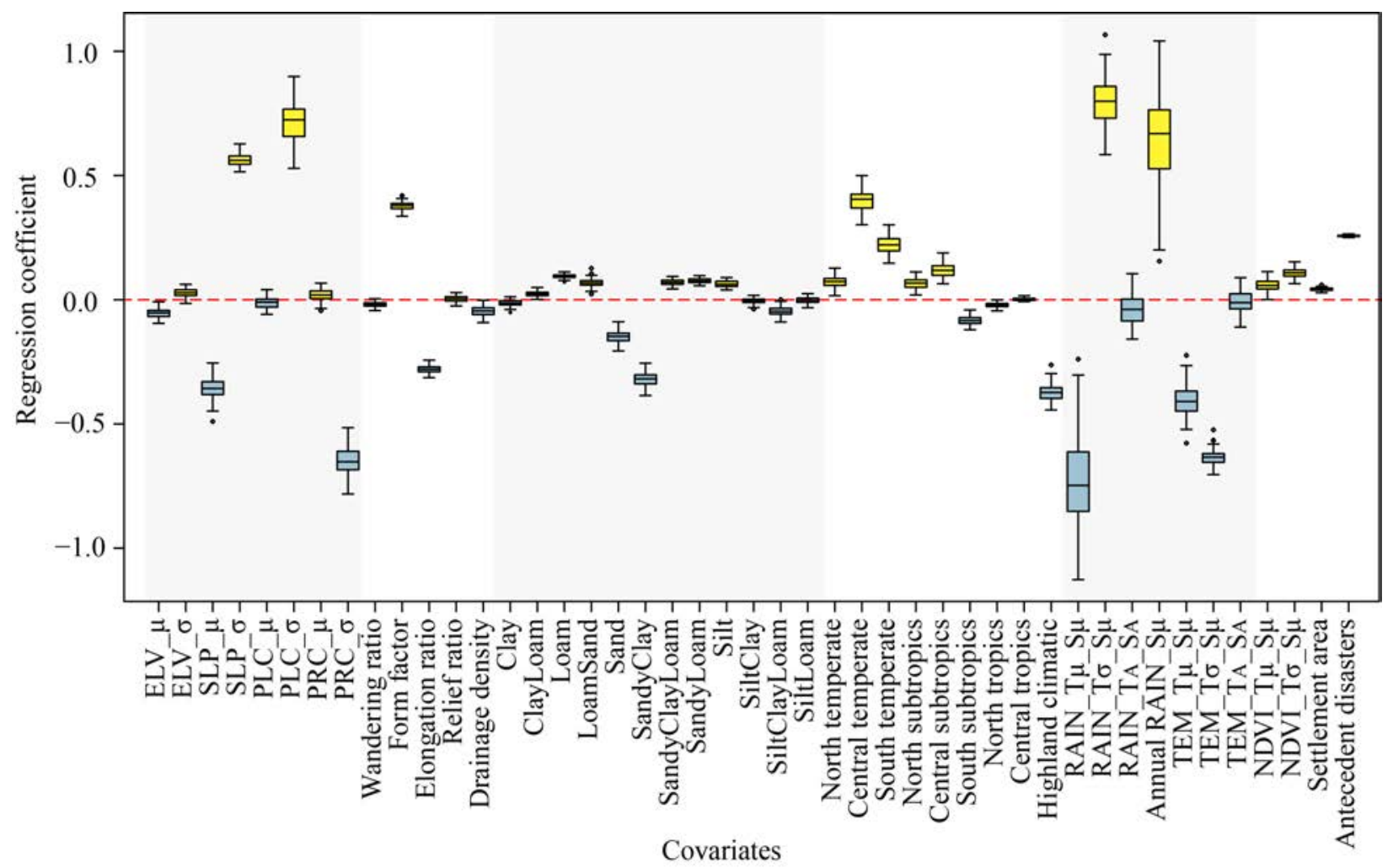

Figure 3: Regression coefficients estimated through the explanatory model built by using the whole HMP spatio-temporal information across China. The covariates shown in this figure are continuous in nature. The red dash line corresponds to zero or no-contribution to the model. Boxplots shown in blue indicate a median negative correlation to HMPs while yellow indicates a median positive one. 


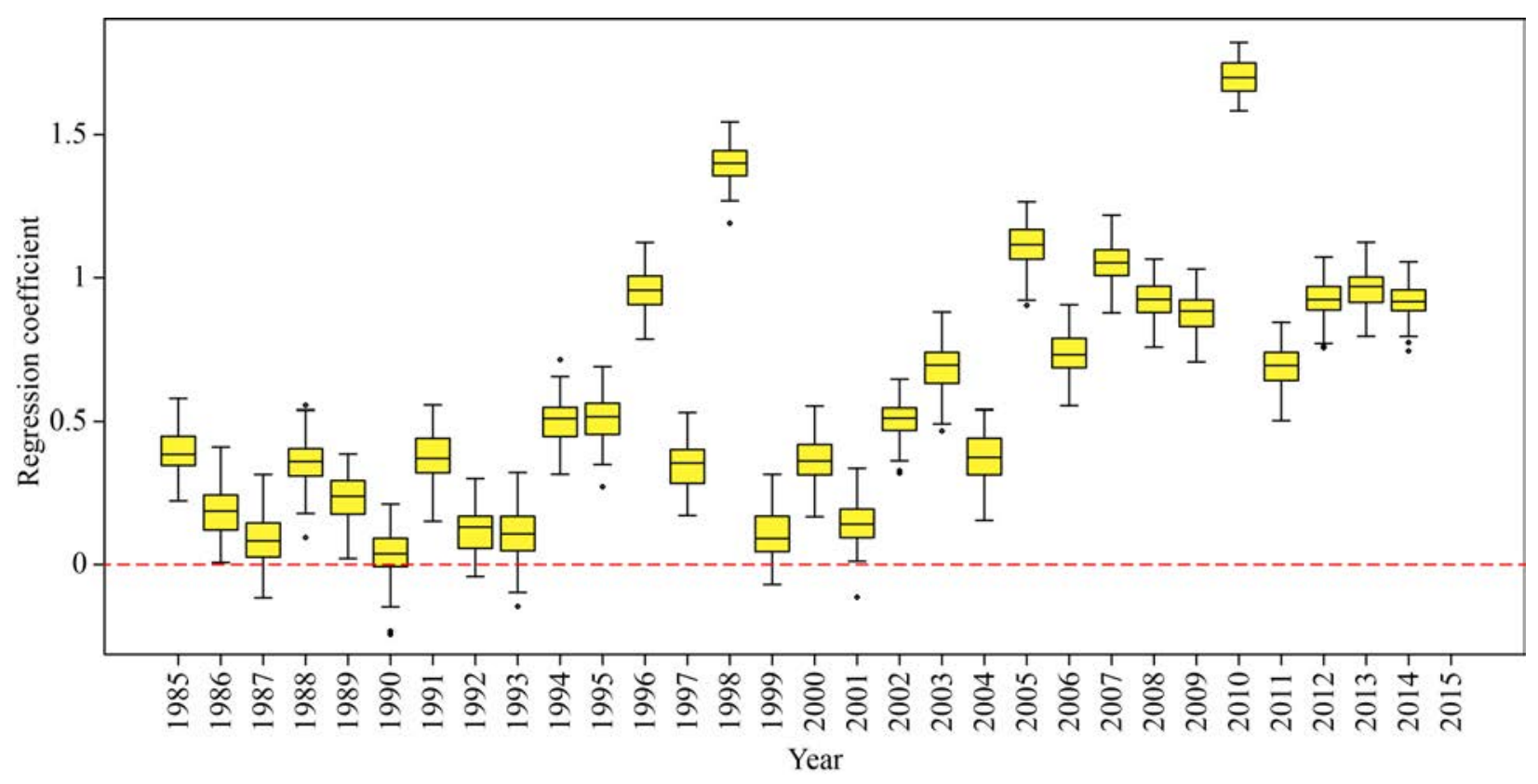

Figure 4: Regression coefficients estimated through the explanatory model built by using the whole HMP spatio-temporal information across China. The covariates shown in this figure are categorical in nature and correspond to the yearly contribution to the model. The red dash line corresponds to zero or no-contribution to the model.
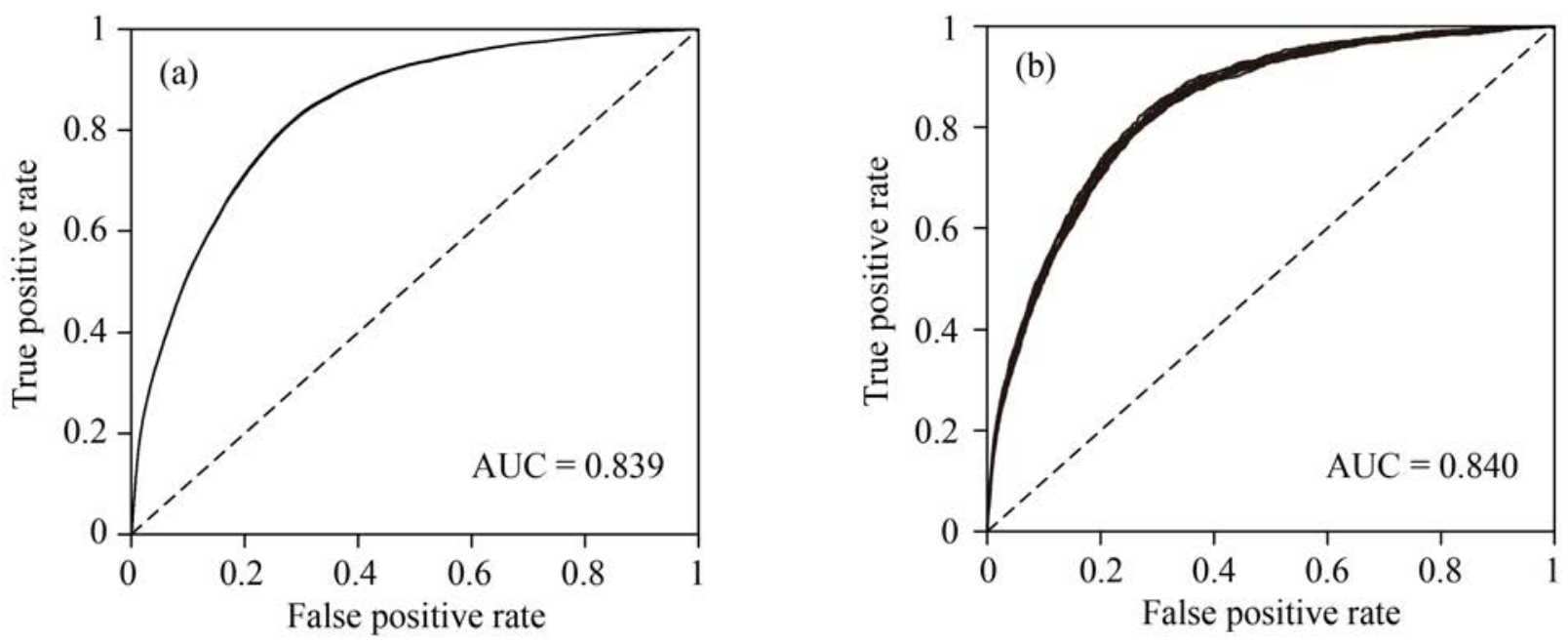

Figure 5: ROC curves obtained via 10-fold cross-validation. (a) Ten calibration models (90\%), (b) Ten validation models (10\%). The AUC reported in both panels corresponds to the mean of the ten replicates, respectively. 


\subsection{Temporal Validation Routines}

Here we present the four temporal validation schemes described in Section 3.5. For each temporal validation scheme, we summarize the model performance in Figure 6. All models are reported with a mean temporal AUC greater than 0.82. We recall here that this value corresponds to excellent performance according to the AUC classification system proposed by Hosmer and Lemeshow (2000). However, two distinct patterns arise in the four temporal validation routines. The AUCs obtained for each year in MOD1 and MOD3 appear quite smooth. In MOD1, this is also associated with a downward shift in AUC when comparing calibration and validation performances (Figure 6a). As for MOD3, calibration and validation performance largely overlap, with the exception of the period in between 2009 and 2015 where the validation routine shows a significant drop in predictive capacity (Figure 6c). In case of MOD2 and MOD4, the AUC values estimated for each year present a much rougher temporal variation. Between these two validation schemes, MOD4 less accurately predicts the HMPs in the last years of our AUC time series (Figure 6d). As for MOD2, a similar difference in performance between calibration and validation is shown for the initial years of our HMP time series (Figure 6b). However, the initial years from 1986 to 1989 contain less HMP occurrences, thus a relatively low performance in this period is much more acceptable than a relatively low performance in the latest years. In light of these considerations, and because of a slightly better performance overall, we consider MOD2 (or Forward-Sequence) as the best validation scheme compared to the other three.

We stress again that a close look at MOD2 in Figure 6b highlights some fluctuations in the AUC time series for the validation whereas the calibration appears much more stable through time in terms of estimated performance. This is better presented in Figure 7 where we show 30 ROC curves, one for each year. The panel (a) corresponds to the training ROC curves and aside for a few years, they consistently overlap through time. As for the validation shown in panel (b) a marked spread can be seen in the curves spanning from 1986 to 2015. We note here that the relatively poorer performance registered at the start and end of the time series also correspond to two years with a relatively lower number of observations. Conversely, the other relatively low AUC values between the two endpoints always appear in the following year containing very large numbers of HMP occurrences. This may be due to the fact that an abrupt increase in HMPs, may induce some variations in the estimated correlations between HMPs and covariates. This in turn, may also induce variations in the susceptibility patterns, which may end up not matching the HMPs of the subsequent year (likely to be representative or closer to the average Chinese susceptibility pattern). This explanation fits well the year 1998. That year was characterized by an exceptionally large number of HMPs in China, and the temporal validation of 1999 returned the poorest performance we observed across the whole temporal sequence. Notably, such temporal variations in performance has been similarly shown in other studies, where the authors reported that effect of climate change may be responsible for large uncertainties in the prediction of HMPs (e.g., Collier, 2007).

To provide a comprehensive overview of the model structure and covariates' role in the 

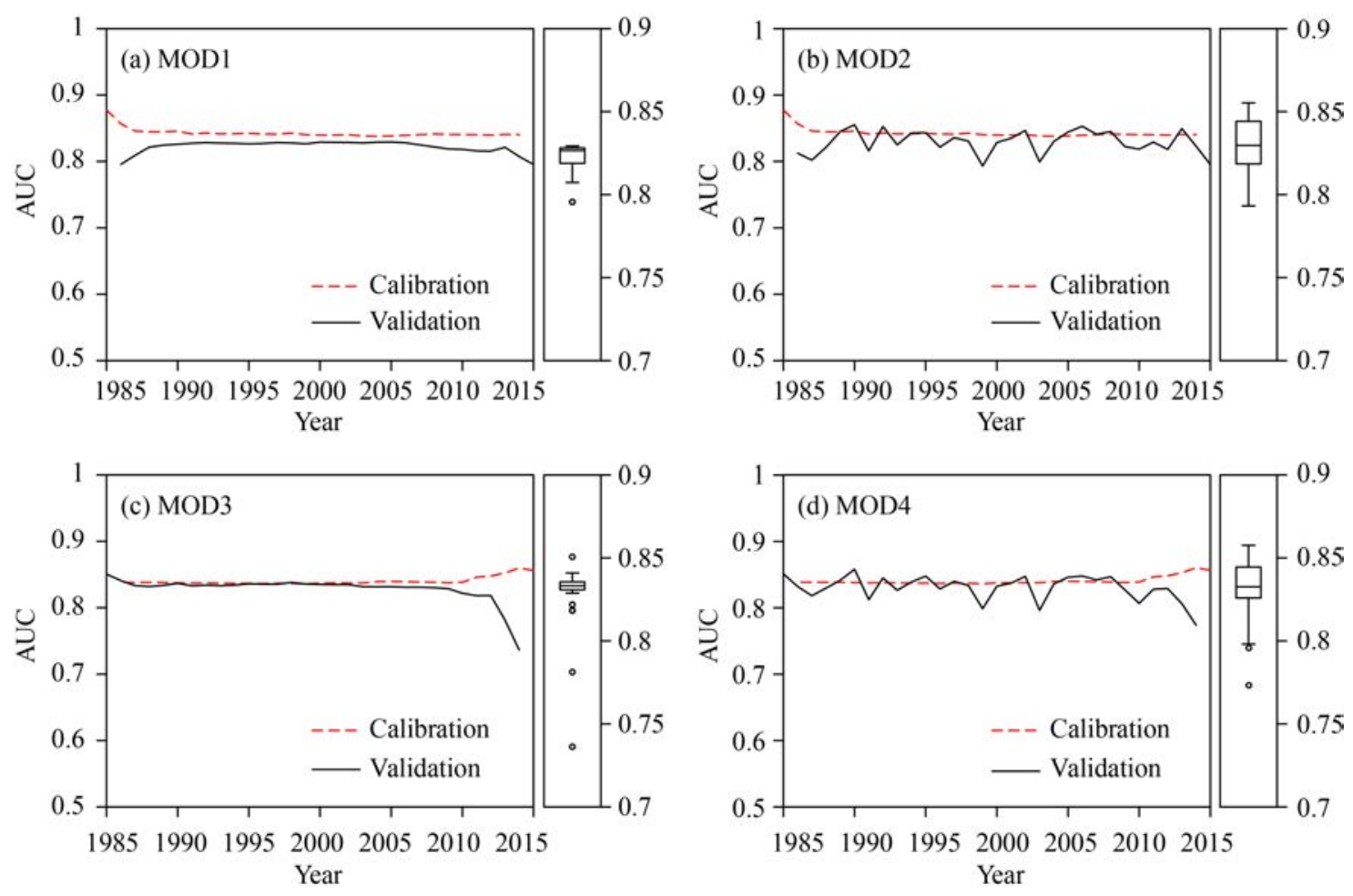

Figure 6: Each panel corresponds to one of the four temporal validations we tested. The line plots report the AUC time series from 1985 to 2015. The boxplots summarize the AUC variation over the thirty years.
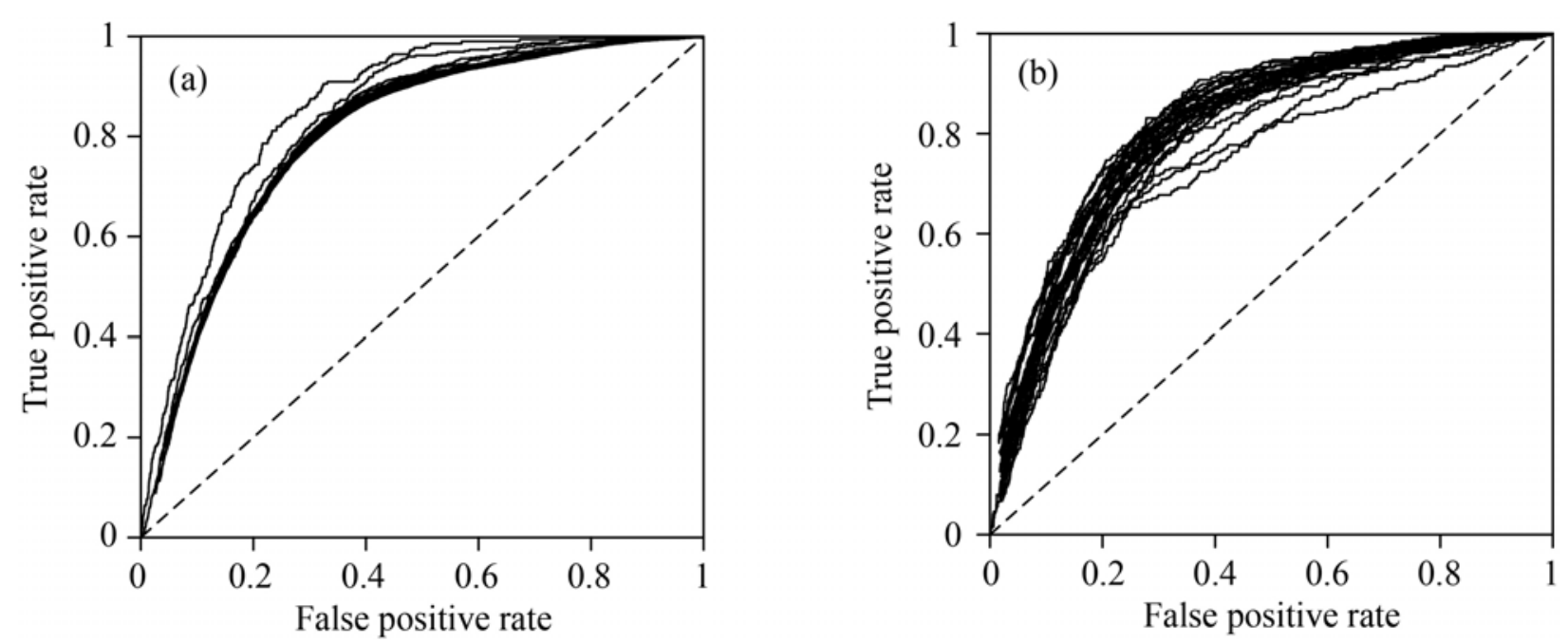

Figure 7: The ROC curves obtained by MOD2. (a) Calibration model, (b) Validation model 
temporal validation, we performed a suite of Jackknife tests (Jiao et al., 2019). We recall here that Jackknife tests are essentially replicates of a reference model whose structure is perturbed by either building single-variable (only-one-variable) models for each of the covariate in the reference structure. Or, by removing one covariate at a time (all-but-onevariable) from the whole set of covariates. Many example of Jackknife tests exist in the susceptibility literature, but they have been limited so far to a pure spatial domain (see for instance, Park, 2015; Lombardo et al., 2016; Ramos-Bernal et al., 2019). Here, because we consider both spatial and temporal dimensions, we iterated the only-one-variable and all-but-one-variable models thirty times, once per year from 1985 to 2015.

Figure 8a presents the AUC obtained via only-one-variable models. It indicates that most of the terrain attributes, climatic indices, and antecedent disasters could contribute to a model with an AUC greater than 0.6. At the same time, the all-but-one-variable models in Figure 8b indicates that removing either of $S L P \_\sigma$, form factor, elongation ratio, $R A I N_{-} T \sigma_{-} S \mu$, and antecedent disasters from the model will induce an obvious AUC drop.

\subsection{Regression Coefficients}

In addition to assessing model performance, another crucial step in any modeling protocol is to evaluate how reasonable regression coefficients may be from an interpretative standpoint. In this work, we already summarized a similar information for our benchmark fit. Nevertheless, regression coefficients estimated for the temporal validation scheme could shed light on the variability that each covariate effect may exhibit through time. Here, we assigned the yellow color for a positive $\beta$ value, which indicates the probability of HMP occurrence will increase by a factor equal to the exponential of the $\beta$ value. Conversely, the blue color indicates a decrease.

Among the terrain attributes, the standard deviation of slope $\left(S L P_{-} \sigma\right)$ and plan curvature $\left(P L C_{-} \sigma\right)$ play an important role in controlling the estimated probability of HMP occurrences (Figure 9). In terms of catchment morphology, form factor and elongation ratio show a positive effect. Most soil types present non significant and negligible contributions to the thirty cross validation schemes, with the exception of Sandy Clay which appears to be negatively correlated to HMPs, although with a slight negative influence. Furthermore, catchments located in Central temperate, South temperate, and Central subtropics zones appear to be more prone to HMPs than the others.

The summary presented above reports the role of time-invariant properties. As for timevariant covariates, AnnualRain_S $\mu$ showed the largest significant and positive effect out of all the climatic indices, followed by $R A I N_{-} T \sigma_{-} \mu$ (the intra-annual rainfall variance within a given catchment). The 3-years antecedent disasters in a given catchment also appeared to be significant and to increase the susceptibility estimates.

Notably, the summary of the covariates' effects shown above is quite static as it overlooks the temporal variation that each model component exhibit as the temporal-validation is performed. To complement this information, in Figure 10 we show the temporal evolution of 
AUC
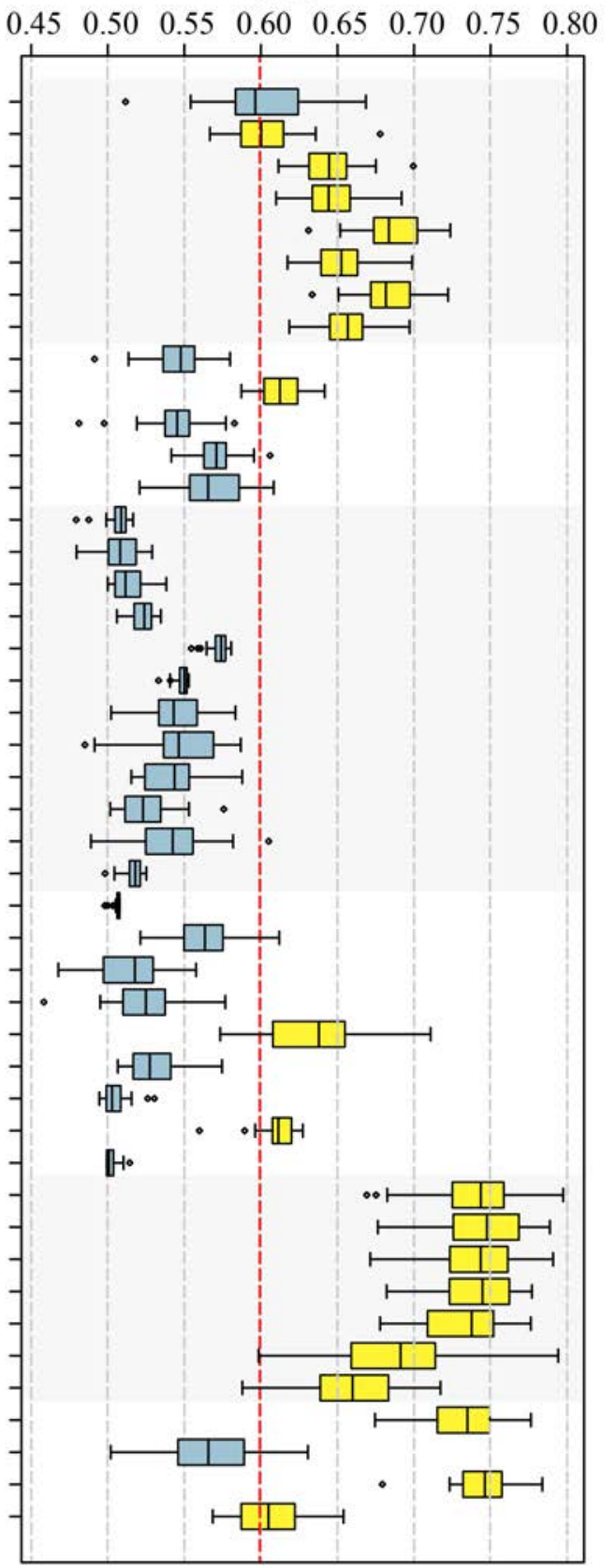

(a) Only-one-variable models

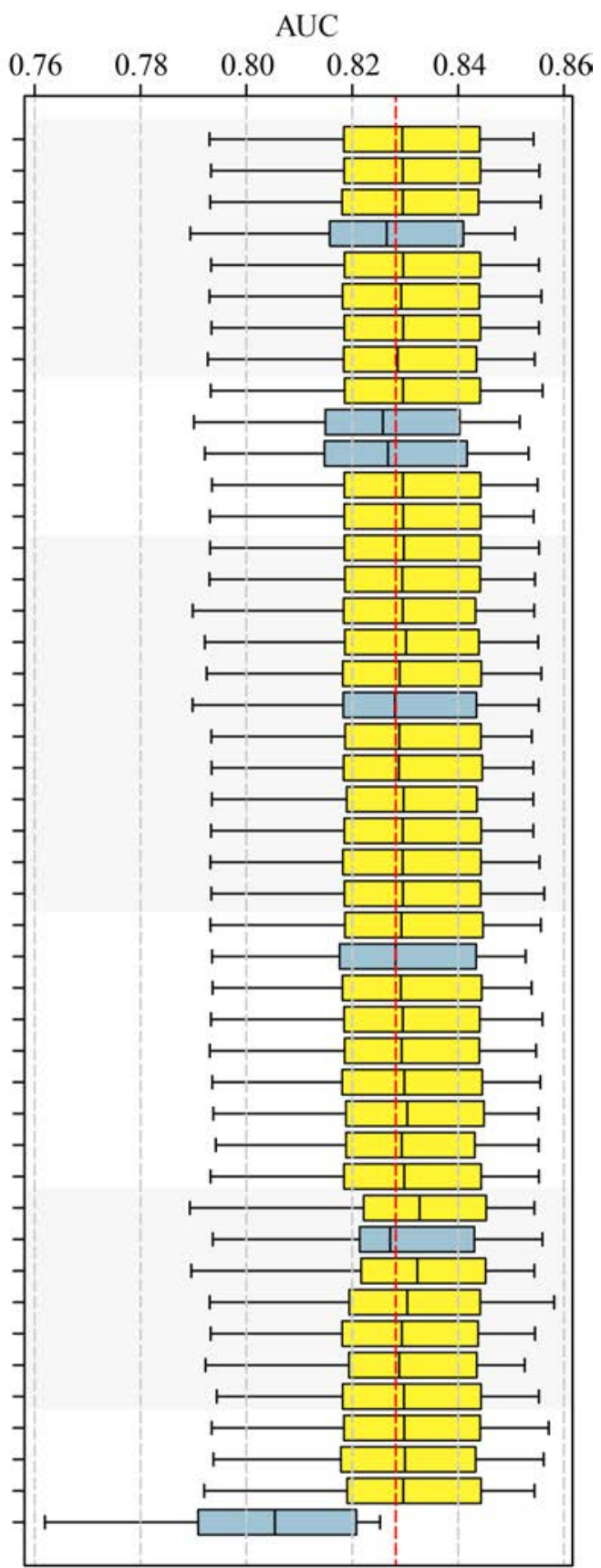

(b) All-but-one-variable models

Figure 8: Jackknife test for covariates. Only-one variable models are shown in the left panel and all-but-one variable models in the right panel. Red line indicates the corresponding mean value of all combinations. Blue boxplots indicate a covariate-specific median AUC lower than the mean AUC computed for all covariates. Yellow boxplots correspond to higher covariate-specific median AUC. 


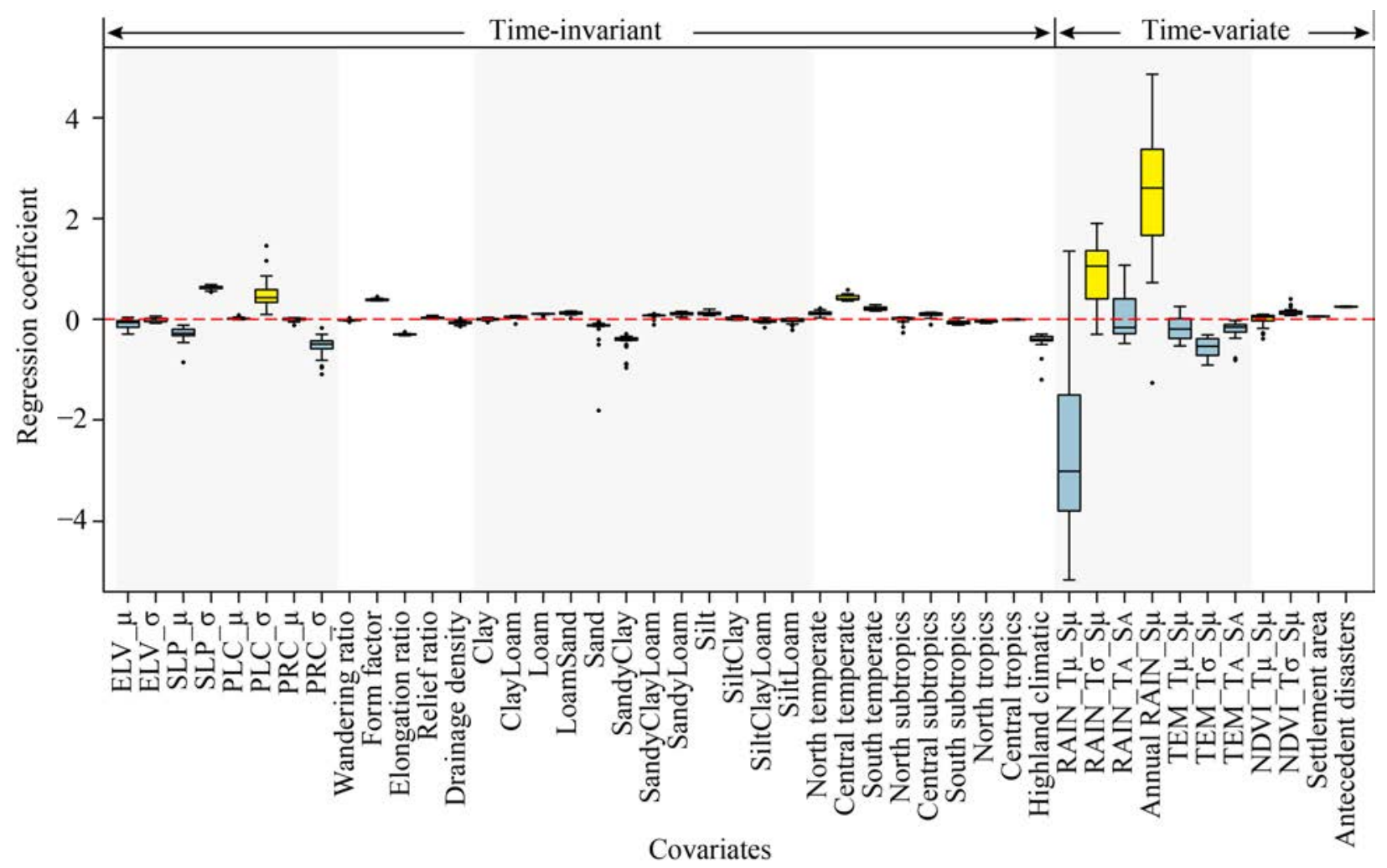

Figure 9: Regression coefficient obtained by MOD2.

the regression coefficients belonging to covariates that appeared to be significant in Figure 9.

More specifically, to better distinguish the variance of the covariates' effects through time, we split Figure 10 in two panels, according to the magnitude of the regression coefficients. Panel (a) summarizes $\beta$ coefficients whose magnitude through time ranges from -0.5 to 0.5 , whereas panel (b) presents the same information for $\beta$ coefficients whose magnitude through time ranges from -5 to 5 . Most of the covariates in both panels indicated a constantly similar effect on HMP occurrence, whereas, few covariates showed a large variation through time. For instance, the annual rainfall (AnnualRAIN_S $N_{-}$) indicated an increasing positive influence from 1985 to 2014. However, the variance of NDVI $\left(N D V I_{-} T \sigma_{-} S \mu\right)$ within each year showed a decreasing effect before 1990, after which the trend flattened until the end of the time series. Overall, the covariates which exhibited the largest variation through time all correspond to climatic indices, especially those associated with rainfall (see AnnualRAIN_S $S$ and $R A I N_{-} T \sigma_{-} S \mu$ in Figure 10b).

\subsection{Susceptibility Mapping}

HMPs susceptibility maps generated via MOD2 are drawn in Figure 11 from 1996 to 2015. These have been classified into very low (VL), low (L), low to medium (LM), medium to 

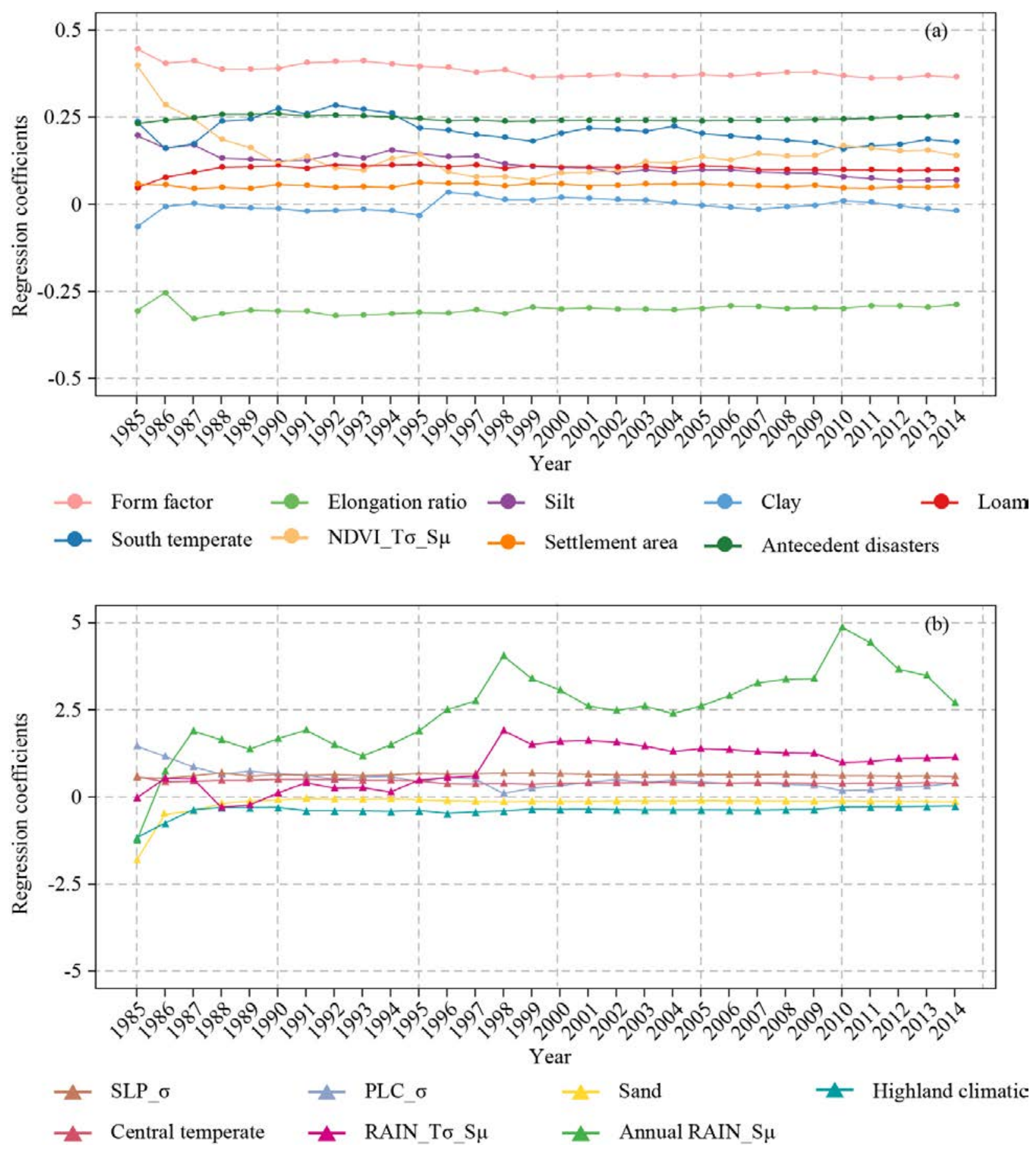

Figure 10: The regression coefficient varying across the of 1986-2015 obtained by MOD2. 
high $(\mathrm{MH})$, high $(\mathrm{H})$, and very high $(\mathrm{VH})$ according to break points that have been set as the $2.5 \%, 25 \%, 50 \%, 75 \%$, and $97.5 \%$ percentiles of the whole probability spectrum combined. In other words, to reclassify the numerical susceptibility into classes, we have concatenated all the space-time HMP probability estimates into a single vector, from which five percentiles have been extracted to ensure a common color palette among the 30 maps.

Looking at the time series of susceptibility maps (Figure 11), at the beginning of the study period probabilities are generally lower, especially in the western sector. Besides, as the time series evolves towards recent years, the probability spectrum appears to shift towards higher susceptibility classes. More specifically, catchments with very low probabilities of HMP occurrences mainly appear from 1986 to 1988; whereas catchments presenting very high probability of HMP occurrences characterize the south-east sector of China since 1997.

To summarize the space-time susceptibility information depicted in Figure 11, we further generated three maps aimed at delivering the mean and the maximum susceptibility together with the $95 \%$ confidence interval (see Figures $12 \mathrm{a}, 12 \mathrm{~b}$ and $12 \mathrm{c}$ respectively).

Looking at the susceptibility patterns depicted in the mean and maximum maps, two macro-regions stand out the most. The western sector of China appears to be consistently estimated as non susceptible. There, the susceptibility appears to be generally confined within the first $10 \%$ of the national distribution. On the contrary, the south-eastern sector appears to be generally the most susceptible. There, most of the catchments are associated with susceptibilities estimated above $70 \%$ of the national probability distribution. Notably, few exceptions exist to this observation due to the existence of large plains, where catchments are generally gentler in topography. Other catchments with high HMP susceptibility, albeit lower than the south-east, can still be found in central, north-east and southern China.

Interestingly, the $95 \%$ confidence interval map - we recall here to be computed as the difference between the $97.5^{\text {th }}$ and $2.5^{\text {th }}$ percentiles of the spatio-temporal probability spectrum shown in Figure 11 - marks analogous geographic patterns to the mean and maximum maps. This is an indication of the robustness of our model. In fact, this means that areas with low susceptibilities are estimated with similar values through time. Conversely, areas with high susceptibility exhibit a much larger degree of variation through time, as expected just by looking at the raw data in Figure 1.

The last panel of Figure 12 depicts seven cluster drawn from the maximum susceptibility in the same figure. These have been manually interpreted on the basis of expert opinion. Clusters $I$ to $V$ correspond to regions are affected by monsoon. The reason to split I and II are due to the difference of terrain and annual rainfall whereas the reason to split I and III into two parts is due to the mountain range that acts as a strong topographic barrier. More specifically:

- Cluster $I$ : the region with the most severe erosion due to the topographic control;

- Cluster $I I$ : the region mostly affected by monsoon;

- Cluster III: less annual rainfall, Loess Plateau affected by widespread gully incisions; 


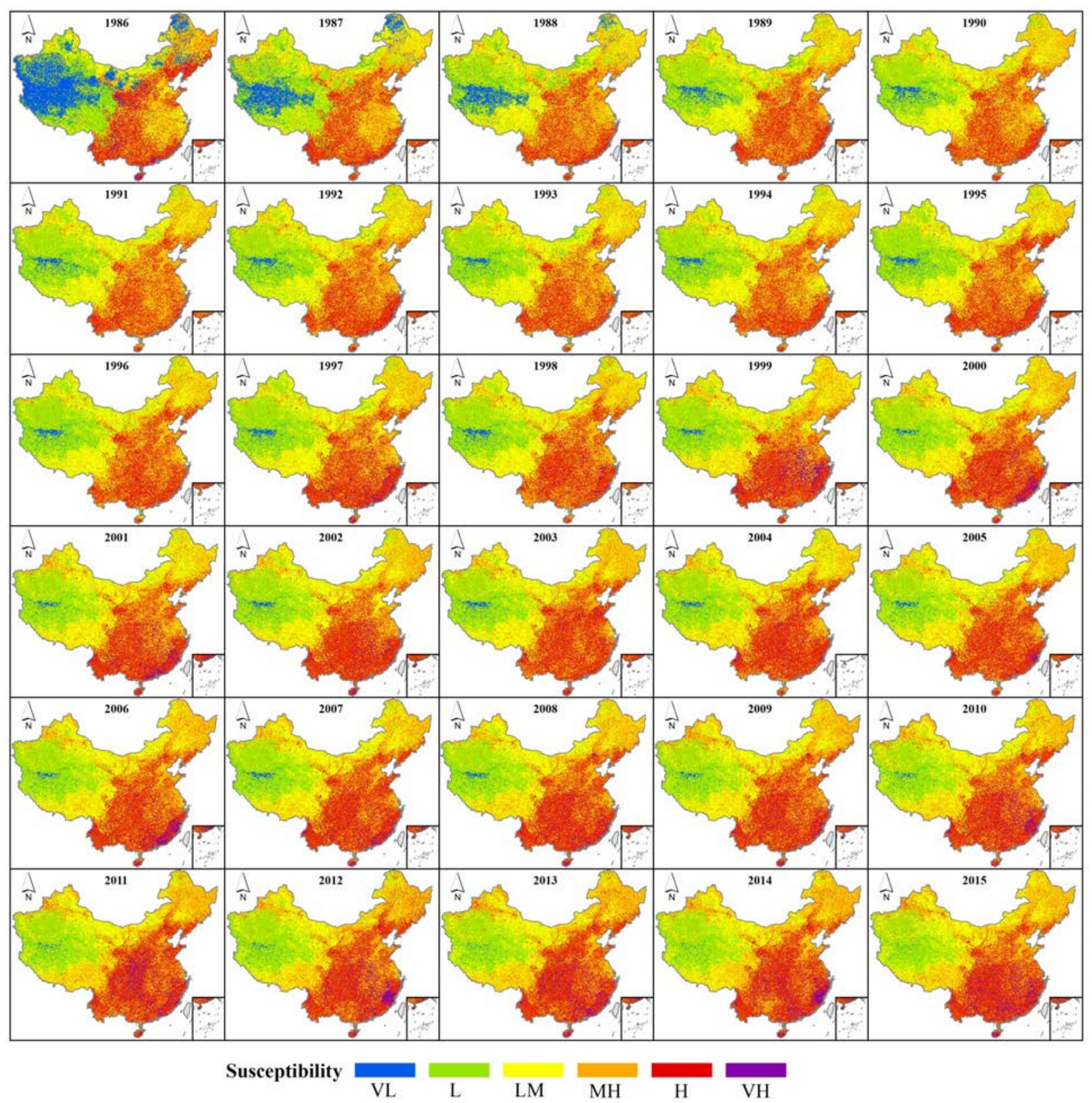

Figure 11: Susceptibility of HMPs in China during 1986-2015 detected via MOD2. 

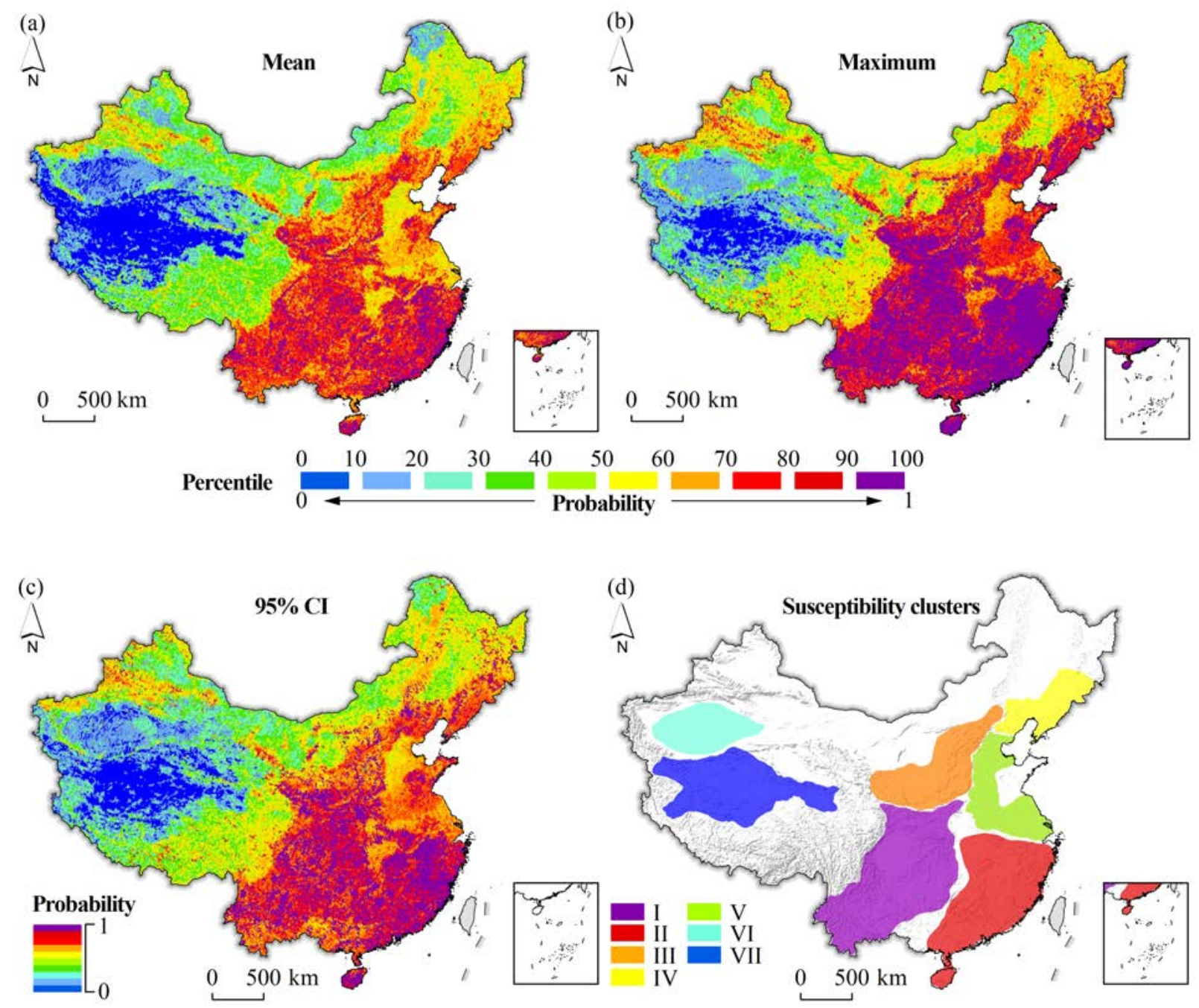

Figure 12: Summary of HMP susceptibility estimated for China from 1986 to 2015 via MOD2: (a) Mean susceptibility, (b) Maximum susceptibility, (c) $95 \%$ CI susceptibility. Panel (d) shows seven interpreted clusters from panel (b). 
- Cluster $I V$ : this sector of China shows a relatively large proneness towards HMP although the rainfall intensity due to the incoming monsoons in this area is much lower than the precipitation discharged to the south. This is primarily due to the local rough topography which contributes to increase the probability of HMP occurrence;

- Cluster $V$ : plains with widespread flat terrains;

- Cluster VI: distinct characteristics attributable to the Taklamakan Desert and the Tarim Basin;

- Cluster VII: sparsely populated area corresponding to the Changtang Plateau and Qinghai Hoh Xil Plateau.

Figure 12 is meant to compress the spatio-temporal susceptibility information in the geographic space. To do the same for the temporal case, we went back to Figure 11 and computed the for each year the areas assigned with one of the six susceptibility class. From these, we generated a stacked barplot (see Figure 13) reporting the proportion of China associated with one of the six classes, showing the evolution through time from 1986 to 2015. What stands out the most is that the areal percentage of catchments with very low (VL) susceptibility decreased sharply in the first three years. This effect is mostly due to the fact that as the time series progressed, more HMP have been recorded, which generally leads to a higher probability of HMP. On the opposite side of the probability spectrum, the proportion of China associated with very high (VH) HMP susceptibility can be seen to have increased from 1997 onward. We remind here the reader that despite these changes may appear small in a simple graphical summary such as Figure 13, in reality a variation of even just $1 \%$ of the total Chinese territory involves several hundreds thousands of $\mathrm{km}^{2}$ and several hundreds actual catchments.

\section{Discussion}

\subsection{Supporting arguments}

This work estimates and investigates the spatio-temporal variation of HMP susceptibility patterns over China. Because of the vast space-time domain, many options exists on how to build and validate a space-time predictive model (Lombardo et al., 2020).

We chose a binomial GLM, which we calibrated and validated through different strategies. The first strategy we used exploited the whole space-time domain, from which catchments with high variations in slope steepness and planar curvature appear to increase the overall susceptibility to HMPs. The influence of slope with respect to HMPs is widely acknowledged in the literature. However, for analyses expressed at the catchment scale, the effect of the terrain steepness may be lost. This may be the reason why in our model, the positive role of the slope steepness is expressed in terms of standard variation, a common proxy for 


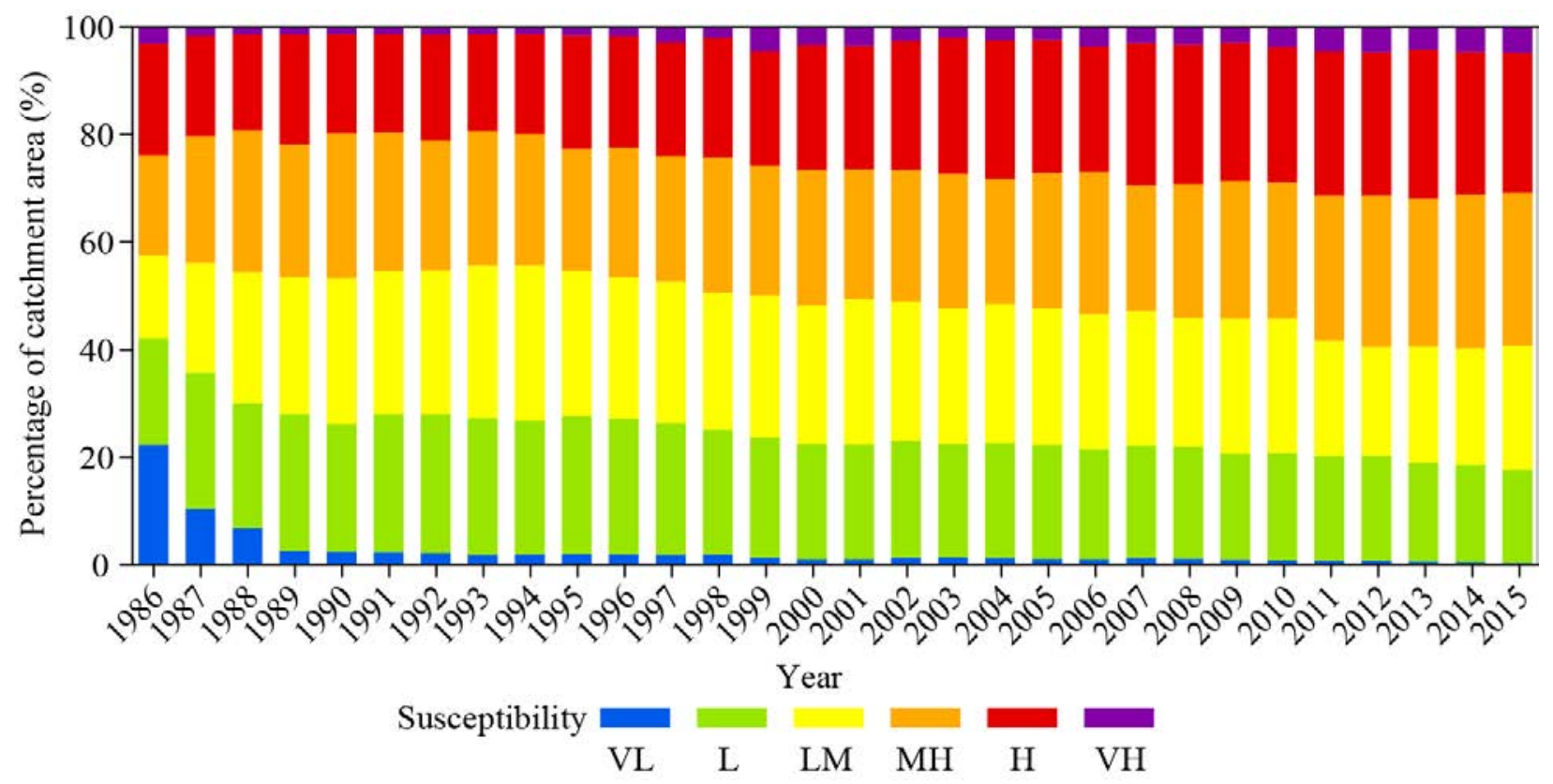

Figure 13: Proportion of the Chinese territory estimated to be HMP-susceptible to HMP, from 1986 to 2015, via MOD2.

topographic roughness. A similar reasoning can be inferred for the standard deviation of the planar curvature.

Unsurprisingly, another positive contribution is carried by the rainfall patterns, expressed through the RAIN_T $\sigma_{-} S \mu$ and the AnnualRAIN_S $\mu$ (see Figure 3). It should be noted that the spatio-temporal rainfall signal is carried in the model via four summary statistics of the precipitation over the mapping (catchment) and over the temporal (year) units. This is certainly the reason behind the overall negative contribution estimated for $R A I N_{-} T \mu_{-} S \mu$. In fact, in any multivariate analysis, whenever slightly dependent covariates interact with each other, the estimation of their sign and amplitude can also depend on each other presence within the model. Because the four rainfall aggregation measures stem from the same spatiotemporal information, it is safe to assume that some degree of dependence can exist among the four we computed. Therefore, the overall influence of rainfall on HMP occurrences should be interpreted as the combined effect of the four covariates and their estimated regression coefficients, which returns an overall increasing effect of the HMP susceptibility as the rainfall increases (see Figure 3 and note the following median values: $\beta_{R A I N_{-} T \mu_{-} S \mu}=$ $\left.-0.75, \beta_{R A I N \_T \sigma-S \mu}=0.80, \beta_{R A I N-T_{A} S_{A}}=-0.04, \beta_{\text {AnnualRAIN_S } \mu}=0.67\right)$.

As for the temperature, the effect is much clearer there, as all the three summary statistics derived from the original spatio-temporal temperature signal appear to have a negative contribution to the model. This means that at increasing temperatures the probability of HMP occurrences consistently decreases in space and time, irrespective of the three components at hand.

We also stress here the relevance of antecedent 3-years disasters. This idea stems from the 
fact that certain types of hazard persist or cluster both in time and space, hence by featuring antecedent occurrences in the model can help predicting future HMPs. This concept is not new in landslide studies (Samia et al., 2018, 2020), although a similar approach has not been tested yet when modeling HMPs statistically.

An additional and equally interesting contribution to the model was carried by human interference. Other researchers have already pointed out a similar consideration (Bronstert, 2003; Plate, 2002), which we tested in this work by including the presence of build-up area per catchment and per year (Marconcini et al., 2020b). The expansion of human settlements has a dual effect in our model. On the one hand, it undeniably constitutes an interference with the environment, potentially leading to HMP occurrences (Duncan, 2013). On the other hand, human expansion also means that a larger number of people are being exposed to disasters (Cutter et al., 2018). This in turns may bring some degree of bias in the HMP inventory because events that occur in non-inhabited areas may not be recorded, especially due to the size of the study area. Conversely, events that occur in inhabited areas are much more likely to be recorded.

As regards the temporal validation scheme we tested, it is important to justify why we chose Mod2 as our best and further presented it to the readers. When looking at performance, not only the central tendency (mean or median) but also the variation around it constitute a relevant criterion. The variation is essentially described as the difference between the highest and lowest performance. Among the two terms, we chose the lowest performance, together with the median AUC, to be our primary mean of selecting the best temporal validation scheme. In fact, in an ideal situation one should avoid selecting a model that can poorly perform even as rare as this may occur. Therefore, MOD2 has become our best validation scheme for it both provides a median value comparable to MOD1, MOD3 and MOD4. And, it returned a minimum AUC much higher than the other temporal validation routines.

In terms of covariates' influence on HMP susceptibility, MOD2 offers a slightly different perspective than the first exploratory tests. The morphological characteristics of the catchments largely contribute to the HMP susceptibility (see form factor and elongation ratio in Figure 10. And even more interestingly, $R A I N_{-} T_{\sigma} S_{\mu}$ and AnnualRAIN $S_{\mu}$ not only dominate the probability estimates to a much larger extent than any other covariate. But, they also show a quite distinctive increasing trend through time.

Ultimately, we decided to remind the reader that the susceptibility we estimated for the whole Chinese territory is actually much finer in resolution than what it looks like in the previous figures. To do this, we have selected eight important and large catchments. In Figure 14 we show the HMP susceptibility estimated for each of those catchments via MOD2, and specifically through the maximum probability of HMP occurrence shown in Figure 12. Looking at Figure 14 becomes evident that our model is built on a very detailed spatial partition of the Chinese landscape. And, within each of the eight selected major catchments, it is possible to further distinguish susceptible sub-catchments that upon which local administrations can made decisions to ensure the safety of local inhabitants. 


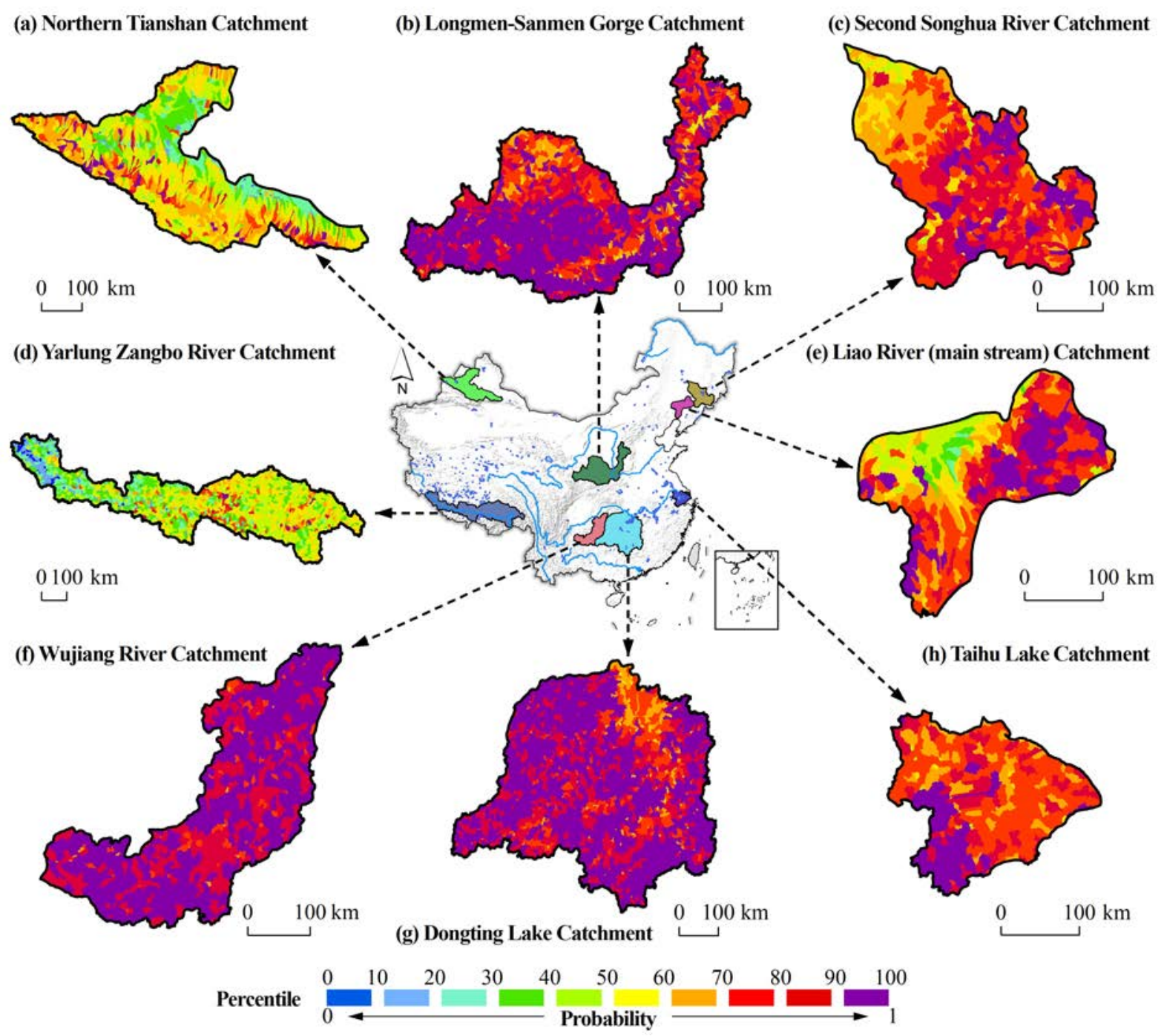

Figure 14: Details of specific large catchments across the Chinese territory. The HMP susceptibility corresponds to the maximum probability estimated via MOD2 between 1986 and 2015, this being shown in Figure 12. The catchments we report here for graphical purposes are: (a) Northern Tianshan Catchment; (b) Longmen-Sanmen Gorge Catchment; (c) Second Songhua River Catchment; (d) Yarlung Zangbo River Catchment; (e) Liao River (main stream) Catchment; (f) Wujiang River Catchment; (g) Dongting Lake Catchment; (h) Taihu Lake Catchment. 


\subsection{Opposing arguments}

The model we present is both spatial and temporal in nature. Among the suitable spacetime models we have chosen a relatively simple one, a binomial GLM. Instead of this, we could have opted for a binomial GAM (Generalized Additive Model) extension to account for possible nonlinear covariates' effects. And, to include potential variables acting at a latent level, hence requiring complex CAR or SPDE components to be featured as well. We maintain that our choice has proven to be a valid option, for both our spatio-temporal cross-validation and temporal validation schemes returned AUC values well above 0.8 , the threshold for excellent binary classifiers according to Hosmer and Lemeshow (2000).

Some may argue that on a 30-year long record, the accuracy of the inventory may have drastically changed in recent times. As a result, the inventory may be biased towards a larger number of HMP records at the end of the time series. We maintain that the HMP inventory is reliable and should not be affected by this type of bias. In fact, the Chinese government has supported the initiative of creating this inventory long before the 1980'ies. And, by the starting year of our time series (1985), the recording protocol had already been standardised at the whole Chinese territory scale. Surely, we cannot entirely disregard the possibility of some sort of bias due to the size of the study area. We know for a fact (and already shared the information with the readers in Section 4) that the western sector of the Chinese territory is devoid of large settlements. This may imply that the lack of HMP record in the region and the subsequent low susceptibility estimated there (see Figures 11 and 12) could be due (to some extent) to a lack of interest rather than a real absence of HMPs. We have tried to investigate this potential issue, by checking local news and other source of information. But, we have not found records of HMPs in the region. Thus, we can only assume the inventory to be reliable.

The spatio-temporal dataset we have built features a much larger number of catchments without HMP records rather than catchments recorded with actual HMP events. In other words, we used an unbalanced presence/absence dataset. In turn, this affects the estimated probability spectrum, resulting in a positively skewed susceptibility distribution. We maintain that this is a realistic pattern for such a vast spatiotemporal domain where probabilities of HMP are generally very low, with the exception of few catchments that are very susceptible (see Frattini et al., 2010). However, it is worth mentioning that the geomorphological community often operates with a balanced dataset of presence and absence cases. This in turn makes the probability spectrum much more normally-distributed and centered at around 0.5 (Rossi et al., 2010). Both approaches are valid, although creating a balanced presence/absence dataset distorts the global intercept (Lombardo and Mai, 2018) making the interpretation of the probabilities valid only in a relative sense rather than the common notion of probability available in any other statistical application (Petschko et al., 2014). Therefore, we maintain that our unbalanced choice is valid and suitable to treat such a large spatiotemporal domain. 


\section{Conclusions}

The Chinese territory hosts a vast and diverse landscape that in the last thirty years has been struck by thousands of hydro-morphological processes. Such processes, spanning from debris flows to debris floods and floods have been monitored and recorded in a multi-temporal digital archive thanks to a Chinese program centrally coordinated but enacted by local administrations. In this work, we explore and exploit this archive to produce the first catchment-scale-based HMP susceptibility model of China, from 1985 to 2015.

We distinguished seven macro-regions where the estimated probability of HMP occurrence can be interpreted and explained. The south-eastern regions are the most susceptible to HMPs, primarily because of the monsoon control on precipitation regimes. This observation of a spatial patterns is strictly entwined with the temporal observation that the susceptibility estimates tend to increase in recent years. This may be due to the fact that climatic changes have narrowed the duration of storms and increased their intensity. This is literally a recipe for disasters, especially in the case of HMP.

For transparency, we are sharing the shapefile containing the susceptibility estimates for each year under consideration. Although we cannot share the raw data, the method we propose is certainly reproducible in any other geographic context. For this reason, we consider our work an example of continental-decadal scale HMP susceptibility. We stress here that other examples currently present in the literature have all been built by using a grid-cell partition of space, where each grid-cell has a resolution in the order of kilometers. Therefore, their actual use is hindered by the fact that over several kilometers, the landscape can contextually feature floodplains as well as mountain ridges. Here we presented a multitemporal HMP susceptibility model built at the catchment scale. Therefore, the information we provide is expressed at a scale that respects the geomorphology and hydrology of the phenomena under consideration. We consider this work a first order indicator of catchments under threat though. And, we expect the operational use of this information to be exploited in a second stage, where physically-based models will be run for catchments with a large probability of HMP occurrence.

\section{Acknowledgement}

This work was supported by the China National Flash Flood Disasters Prevention and Control Project. The authors are grateful for financial support from the National Natural Science Foundation of China [grant number No. 41590845], and the China Institute of Water Resources and Hydropower Research (IWHR)[grant number No. SHZH-IWHR-57]. 


\section{References}

Adnan, M. S. G., Dewan, A., Zannat, K. E. and Abdullah, A. Y. M. (2019) The use of watershed geomorphic data in flash flood susceptibility zoning: a case study of the Karnaphuli and Sangu river basins of Bangladesh. Natural Hazards 99(1), 425-448.

Blöschl, G., Kiss, A., Viglione, A., Barriendos, M., Böhm, O., Brázdil, R., Coeur, D., Demarée, G., Llasat, M. C., Macdonald, N. et al. (2020) Current European flood-rich period exceptional compared with past 500 years. Nature 583(7817), 560-566.

Borga, M., Boscolo, P., Zanon, F. and Sangati, M. (2007) Hydrometeorological analysis of the 29 August 2003 flash flood in the Eastern Italian Alps. Journal of Hydrometeorology 8(5), 1049-1067.

Bout, B., Lombardo, L., van Westen, C. and Jetten, V. (2018) Integration of two-phase solid fluid equations in a catchment model for flashfloods, debris flows and shallow slope failures. Environmental Modelling \& Software 105, 1-16.

Bronstert, A. (2003) Floods and climate change: interactions and impacts. Risk Analysis: An International Journal 23(3), 545-557.

Budimir, M., Atkinson, P. and Lewis, H. (2015) A systematic review of landslide probability mapping using logistic regression. Landslides 12(3), 419-436.

Cama, M., Lombardo, L., Conoscenti, C., Agnesi, V. and Rotigliano, E. (2015) Predicting storm-triggered debris flow events: application to the 2009 Ionian Peloritan disaster (Sicily, Italy). Nat Hazards Earth Syst Sci 15(8), 1785-1806.

Carrara, A., Cardinali, M., Detti, R., Guzzetti, F., Pasqui, V. and Reichenbach, P. (1991) GIS techniques and statistical models in evaluating landslide hazard. Earth Surface Processes and Landforms 16(5), 427-445.

Carrara, A., Cardinali, M., Guzzetti, F. and Reichenbach, P. (1995) Gis technology in mapping landslide hazard. In Geographical Information Systems in Assessing Natural Hazards, Advances in Natural and Technological Hazards Research, pp. 135-175. Dordrecht: Kluwer, Springer. ISBN 978-90-481-4561-4 978-94-015-8404-3.

Cenci, L., Laiolo, P., Gabellani, S., Campo, L., Silvestro, F., Delogu, F., Boni, G. and Rudari, R. (2016) Assimilation of H-SAF soil moisture products for flash flood early warning systems. Case study: Mediterranean catchments. IEEE Journal of Selected Topics in Applied Earth Observations and Remote Sensing 9(12), 5634-5646.

Collier, C. (2007) Flash flood forecasting: What are the limits of predictability? Quarterly Journal of the Royal Meteorological Society: A journal of the atmospheric sciences, applied meteorology and physical oceanography 133(622), 3-23. 
Crema, S., Marra, F., Andreoli, A., Scorpio, V., Kofler, C., Cavalli, M., Marchi, L., Borga, M. and Comiti, F. (2018) Integrating high-resolution hydrology and geomorphometry for flash flood characterization. EGUGA p. 13142.

Cutter, S. L., Emrich, C. T., Gall, M. and Reeves, R. (2018) Flash flood risk and the paradox of urban development. Natural Hazards Review 19(1), 05017005.

Duncan, J. M. (2013) Slope stability then and now. In Geo-Congress 2013: Stability and Performance of Slopes and Embankments III, pp. 2184-2203.

Efron, B. and Tibshirani, R. J. (1994) An introduction to the bootstrap. CRC press.

Frattini, P., Crosta, G. and Carrara, A. (2010) Techniques for evaluating the performance of landslide susceptibility models. Engineering Geology 111(1), 62-72.

Gaume, E., Bain, V., Bernardara, P., Newinger, O., Barbuc, M., Bateman, A., Blaškovičová, L., Blöschl, G., Borga, M., Dumitrescu, A. et al. (2009) A compilation of data on European flash floods. Journal of Hydrology 367(1-2), 70-78.

Glade, T. and Crozier, M. J. (2005) Landslide hazard and risk: concluding comment and perspectives. Landslide hazard and risk. Wiley, Chichester pp. 767-774.

Gong, P., Li, X. and Zhang, W. (2019) 40-Year (1978-2017) human settlement changes in China reflected by impervious surfaces from satellite remote sensing. Science Bulletin 64(11), 756-763.

Gourley, J. J., Flamig, Z. L., Vergara, H., Kirstetter, P.-E., Clark III, R. A., Argyle, E., Arthur, A., Martinaitis, S., Terti, G., Erlingis, J. M. et al. (2017) The FLASH Project: improving the tools for flash flood monitoring and prediction across the United States. Bulletin of the American Meteorological Society 98(2), 361-372.

Gourley, J. J., Hong, Y., Flamig, Z. L., Arthur, A., Clark, R., Calianno, M., Ruin, I., Ortel, T., Wieczorek, M. E., Kirstetter, P.-E. et al. (2013) A unified flash flood database across the United States. Bulletin of the American Meteorological Society 94(6), 799-805.

Guo, L., He, B., Ma, M., Chang, Q., Li, Q., Zhang, K. and Hong, Y. (2018) A comprehensive flash flood defense system in China: overview, achievements, and outlook. Natural Hazards 92(2), $727-740$.

Guzzetti, F., Mondini, A. C., Cardinali, M., Fiorucci, F., Santangelo, M. and Chang, K.-T. (2012) Landslide inventory maps: New tools for an old problem. Earth-Science Reviews 112(1-2), 42-66.

Hosmer, D. W. and Lemeshow, S. (2000) Applied Logistic Regression. Second edition. New York: Wiley. 
Javelle, P., Fouchier, C., Arnaud, P. and Lavabre, J. (2010) Flash flood warning at ungauged locations using radar rainfall and antecedent soil moisture estimations. Journal of Hydrology 394(1-2), 267-274.

Jiao, Y., Zhao, D., Ding, Y., Liu, Y., Xu, Q., Qiu, Y., Liu, C., Liu, Z., Zha, Z. and Li, R. (2019) Performance evaluation for four GIS-based models purposed to predict and map landslide susceptibility: A case study at a world heritage site in Southwest China. Catena 183, 104221.

Karagiorgos, K., Thaler, T., Heiser, M., Hübl, J. and Fuchs, S. (2016) Integrated flash flood vulnerability assessment: insights from East Attica, Greece. Journal of Hydrology 541, $553-562$.

Li, H., Lei, X., Shang, Y. and Qin, T. (2018) Flash flood early warning research in China. International Journal of Water Resources Development 34(3), 369-385.

Li, H., Wang, D., Singh, V. P., Wang, Y., Wu, J., Wu, J., Liu, J., Zou, Y., He, R. and Zhang, J. (2019a) Non-stationary frequency analysis of annual extreme rainfall volume and intensity using Archimedean copulas: A case study in eastern China. Journal of Hydrology 571, 114-131.

Li, W., Lin, K., Zhao, T., Lan, T., Chen, X., Du, H. and Chen, H. (2019b) Risk assessment and sensitivity analysis of flash floods in ungauged basins using coupled hydrologic and hydrodynamic models. Journal of Hydrology 572, 108-120.

Liao, Z., Hong, Y., Kirschbaum, D. and Liu, C. (2012) Assessment of shallow landslides from Hurricane Mitch in central America using a physically based model. Environmental Earth Sciences 66(6), 1697-1705.

Liu, C., Guo, L., Ye, L., Zhang, S., Zhao, Y. and Song, T. (2018) A review of advances in China's flash flood early-warning system. Natural Hazards 92(2), 619-634.

Lombardo, L., Bakka, H., Tanyas, H., van Westen, C., Mai, P. M. and Huser, R. (2019) Geostatistical modeling to capture seismic-shaking patterns from earthquake-induced landslides. Journal of Geophysical Research: Earth Surface 124(7), 1958-1980.

Lombardo, L., Cama, M., Conoscenti, C., Märker, M. and Rotigliano, E. (2015) Binary logistic regression versus stochastic gradient boosted decision trees in assessing landslide susceptibility for multiple-occurring landslide events: application to the 2009 storm event in Messina (Sicily, southern Italy). Natural Hazards 79(3), 1621-1648.

Lombardo, L., Fubelli, G., Amato, G. and Bonasera, M. (2016) Presence-only approach to assess landslide triggering-thickness susceptibility: a test for the Mili catchment (northeastern Sicily, Italy). Natural Hazards 84(1), 565-588. 
Lombardo, L. and Mai, P. M. (2018) Presenting logistic regression-based landslide susceptibility results. Engineering geology 244, 14-24.

Lombardo, L., Opitz, T., Ardizzone, F., Guzzetti, F. and Huser, R. (2020) Space-time landslide predictive modelling. Earth-Science Reviews p. 103318.

Lombardo, L. and Tanyas, H. (2020) Chrono-validation of near-real-time landslide susceptibility models via plug-in statistical simulations. Engineering Geology p. 105818.

Mahmood, M. I., Elagib, N. A., Horn, F. and Saad, S. A. (2017) Lessons learned from Khartoum flash flood impacts: An integrated assessment. Science of the Total Environment 601, 1031-1045.

Marchi, L., Borga, M., Preciso, E. and Gaume, E. (2010) Characterisation of selected extreme flash floods in Europe and implications for flood risk management. Journal of Hydrology 394(1-2), 118-133.

Marconcini, M., Gorelick, N., Metz-Marconcini, A. and Esch, T. (2020a) Accurately monitoring urbanization at global scale-the world settlement footprint. In IOP Conference Series: Earth and Environmental Science, volume 509, p. 012036.

Marconcini, M., Metz-Marconcini, A., Üreyen, S., Palacios-Lopez, D., Hanke, W., Bachofer, F., Zeidler, J., Esch, T., Gorelick, N., Kakarla, A. et al. (2020b) Outlining where humans live, the World Settlement Footprint 2015. Scientific Data 7(1), 1-14.

Norbiato, D., Borga, M., Degli Esposti, S., Gaume, E. and Anquetin, S. (2008) Flash flood warning based on rainfall thresholds and soil moisture conditions: An assessment for gauged and ungauged basins. Journal of Hydrology 362(3-4), 274-290.

O'Banion, M. and Olsen, M. (2014) Predictive seismically-induced landslide hazard mapping in oregon using a maximum entropy model (MaxEnt). In Proceedings of the 10th national conference in earthquake engineering, Earthquake Engineering Research Institute, Anchorage.

Paprotny, D., Morales-Nápoles, O. and Jonkman, S. N. (2017) Efficient pan-European river flood hazard modelling through a combination of statistical and physical models. Natural Hazards and Earth System Sciences 17(7), 1267.

Park, N.-W. (2015) Using maximum entropy modeling for landslide susceptibility mapping with multiple geoenvironmental data sets. Environmental Earth Sciences 73(3), 937-949.

Petschko, H., Brenning, A., Bell, R., Goetz, J. and Glade, T. (2014) Assessing the quality of landslide susceptibility maps - case study lower austria. Natural Hazards and Earth System Sciences 14(1), 95-118. 
Plate, E. J. (2002) Flood risk and flood management. Journal of Hydrology 267(1-2), 2-11.

Prein, A. F., Rasmussen, R. M., Ikeda, K., Liu, C., Clark, M. P. and Holland, G. J. (2017) The future intensification of hourly precipitation extremes. Nature Climate Change $\mathbf{7}(1)$, $48-52$.

Ragettli, S., Zhou, J., Wang, H., Liu, C. and Guo, L. (2017) Modeling flash floods in ungauged mountain catchments of China: A decision tree learning approach for parameter regionalization. Journal of Hydrology 555, 330-346.

Rahmati, O., Kornejady, A., Samadi, M., Deo, R. C., Conoscenti, C., Lombardo, L., Dayal, K., Taghizadeh-Mehrjardi, R., Pourghasemi, H. R., Kumar, S. et al. (2019) PMT: New analytical framework for automated evaluation of geo-environmental modelling approaches. Science of the total environment 664, 296-311.

Ramos-Bernal, R. N., Vázquez-Jiménez, R., Tizapa, S. S. and Matus, R. A. (2019) Characterization of Susceptible Landslide Zones by an Accumulated Index. In Landslides. IntechOpen.

Reichenbach, P., Rossi, M., Malamud, B. D., Mihir, M. and Guzzetti, F. (2018) A review of statistically-based landslide susceptibility models. Earth-Science Reviews 180, 60-91.

Rossi, M., Guzzetti, F., Reichenbach, P., Mondini, A. C. and Peruccacci, S. (2010) Optimal landslide susceptibility zonation based on multiple forecasts. Geomorphology 114(3), 129142.

Rossi, M., Guzzetti, F., Salvati, P., Donnini, M., Napolitano, E. and Bianchi, C. (2019) A predictive model of societal landslide risk in Italy. Earth-Science Reviews 196, 102849.

Rozalis, S., Morin, E., Yair, Y. and Price, C. (2010) Flash flood prediction using an uncalibrated hydrological model and radar rainfall data in a Mediterranean watershed under changing hydrological conditions. Journal of Hydrology 394(1-2), 245-255.

Samia, J., Temme, A., Bregt, A., Wallinga, J., Guzzetti, F. and Ardizzone, F. (2020) Dynamic path-dependent landslide susceptibility modelling. Natural Hazards and Earth System Sciences 20(1), 271-285.

Samia, J., Temme, A., Bregt, A. K., Wallinga, J., Stuiver, J., Guzzetti, F., Ardizzone, F. and Rossi, M. (2018) Implementing landslide path dependency in landslide susceptibility modelling. Landslides 15(11), 2129-2144.

Samia, J., Temme, A. J., Bregt, A., Wallinga, J., Guzzetti, F., Ardizzone, F. and Rossi, M. (2017) Do Landslides Follow Landslides? Insights in Path Dependency from a MultiTemporal Landslide Inventory. Landslides 14, 547-558. 
Shen, X., Anagnostou, E. N., Mei, Y. and Hong, Y. (2017) A global distributed basin morphometric dataset. Scientific Data 4(1), 1-8.

Špitalar, M., Gourley, J. J., Lutoff, C., Kirstetter, P.-E., Brilly, M. and Carr, N. (2014) Analysis of flash flood parameters and human impacts in the US from 2006 to 2012. Journal of Hydrology 519, 863-870.

Tramblay, Y., Bouvier, C., Martin, C., Didon-Lescot, J.-F., Todorovik, D. and Domergue, J.-M. (2010) Assessment of initial soil moisture conditions for event-based rainfall-runoff modelling. Journal of Hydrology 387(3-4), 176-187.

Wang, N., Cheng, W., Wang, B., Liu, Q. and Zhou, C. (2020) Geomorphological regionalization theory system and division methodology of China. Journal of Geographical Sciences 30(2), 212-232.

Westra, S., Fowler, H., Evans, J., Alexander, L., Berg, P., Johnson, F., Kendon, E., Lenderink, G. and Roberts, N. (2014) Future changes to the intensity and frequency of short-duration extreme rainfall. Reviews of Geophysics 52(3), 522-555. 